\title{
The Index of Elliptic Operators on Manifolds with Conical Points
}

\author{
Boris Fedosov \\ Bert-Wolfgang Schulze \\ Nikolai Tarkhanov* \\ Institut für Mathematik \\ Universität Potsdam \\ Postfach 601553 \\ 14415 Potsdam \\ Germany
}

August 29, 1997

${ }^{*}$ Supported by the Deutsche Forschungsgemeinschaft. 


\begin{abstract}
For general elliptic pseudodifferential operators on manifolds with singular points, we prove an algebraic index formula. In this formula the symbolic contributions from the interior and from the singular points are explicitly singled out. For two-dimensional manifolds, the interior contribution is reduced to the Atiyah-Singer integral over the cosphere bundle while two additional terms arise. The first of the two is one half of the 'eta' invariant associated to the conormal symbol of the operator at singular points. The second term is also completely determined by the conormal symbol. The example of the Cauchy-Riemann operator on the complex plane shows that all the three terms may be non-zero.
\end{abstract}

AMS subject classification: primary: 58G10; secondary: 58G03.

Key words and phrases: manifolds with singularities, pseudodifferential operators, elliptic operators, index. 


\section{Contents}

Introduction $\quad 5$

1 Formal symbols $\quad 8$

2 Pseudodifferential operators $\quad 12$

3 Algebraic index theorem $\quad 15$

4 Two-dimensional case $\quad 23$

5 Examples $\quad 31$

$\begin{array}{ll}\text { References } & 35\end{array}$ 


\section{Introduction}

Since Atiyah, Patodi and Singer [APS75] investigated the index problem for first order elliptic differential operators arising in Riemannian geometry on manifolds with cylindrical ends and introduced the famous 'eta' invariant, there appeared a great number of papers dealing with index theorems for elliptic operators on manifolds with conical points. Among notable generalisations of their formula in the setting of Dirac operators are those to noncompact manifolds by Brüning and Seeley [BS88], Brüning [Brü90], Müller [Mü187], Stern [Ste89, Ste90] and Klimek and Wojciechowski [KW93], to singular manifolds by Cheeger [Che83, Che87], Melrose [Mel93], Lesch [Les97] and Hassell, Mazzeo and Melrose [HMM96], to boundary value problems by Branson and Gilkey [BG92], Douglas and Wojciechowski [DW91], Müller [Mü194], Grubb [Gru92] and Grubb and Seeley [GS95], to families by Bismut and Cheeger [BC89, BC90] and Melrose and Piazza [MP93], and also to define "higher" 'eta' invariants by Lott [Lot92], Getzler [Get93] and Wu [Wu93].

The problem of extending the Atiyah-Patodi-Singer index theorem to a general index theorem for elliptic pseudodifferential operators on manifolds with conical singularities is of considerable interest. For a class of zero order pseudodifferential operators on a closed manifold having a discontinuity of first kind at isolated points, an index formula was proved by Plamenevskii and Rozenblum [PR90, PR92]. Their approach is based on considering $C^{*}$-algebras generated by such operators; it was further developed by Rozenblum [Roz94]. In the framework of the $b$-calculus of Melrose [Mel81] and Melrose and Mendoza [MM83], an index formula for general elliptic operators was established by Piazza [Pia93] who made use of the 'zeta' function approach. His formula expresses the index of an operator acting on a Sobolev space of weight $\gamma \in \mathbb{R}$, as the sum of an interior contribution given in terms of regularised 'zeta' functions, and a boundary contribution generalising the 'eta' invariant of Atiyah, Patodi and Singer. This second term measures the asymmetry of the spectrum of the indicial operator (or conormal symbol) with respect to a weight line $\Gamma_{\gamma}$ in the complex plane. While the Atiyah-Patodi-Singer index theorem for a twisted Dirac operator can be obtained from the formula of [Pia93], it is still an open problem as to whether, for a general elliptic $b$-pseudodifferential operator $A$, the interior contribution depends only on the principal symbol of $A$. For an elliptic differential operator on a manifold with a conical point whose conormal symbol is symmetric with respect to some weight line, Schulze, Sternin and Shatalov [SSS97] proved a formula expressed the index as the sum of the ranks of spectral points of the conormal symbol and the integral from the Atiyah-Singer form over the smooth part of the manifold. The authors have 
also contributed to this problem.

But despite such an activity during the last 20 years the situation remains still unsatisfactory. The main difficulty is that the algebra of pseudodifferential operators on a manifold with conical singularities consists of components of different nature (interior and conormal) obeying some compatibility conditions, and it is not quite clear in which terms the index of elliptic operators should be expressed. In other words, one has to find proper functionals on this algebra to express the index in a possibly simple way. Such an investigation was done by Melrose and Nistor [MN96a] who computed the Hochschild and cyclic homology groups for the algebra of 'cusp' pseudodifferential operators on any manifold with boundary. These computations are closely related to the index problem since the index functional for this algebra can be interpreted as a Hochschild 1-cocycle. Their index formulas contain various extensions of the trace functional of the Wodzicki non-commutative residue type, thus resulting in a pseudodifferential generalisation of the Atiyah-Patodi-Singer index theorem. In [MN96a], they also computed $K$-theory invariants of algebras of pseudodifferential operators on manifold with corners and proved an equivariant index theorem.

Here we use another approach based on the algebra of formal symbols on a manifold with conical points blown up to a smooth manifold with boundary, $M$. This algebra is a particular case of the algebra of observables in deformation quantisation adopted to the special cotangent bundle structure of the symplectic manifold in question. It was previously used by the first author [Fed74] (see also Section 1 below for a short review) to approximate pseudodifferential operators on a smooth manifold up to trace class operators. It turns out that the algebra of formal symbols is equally good to approximate the conormal components of pseudodifferential operators on manifolds with conical points. This approximation leads to an index formula which we call algebraic,

$$
\text { ind } \begin{aligned}
A & =\left.\operatorname{Tr}(1-\bar{r} \circ \bar{a})\right|_{N}-\left.\operatorname{Tr}(1-\bar{a} \circ \bar{r})\right|_{N} \\
& +\frac{1}{2 \pi i} \int_{-\infty}^{\infty} d \tau \operatorname{Tr}^{\prime}\left(A_{c}^{-1}(\tau+i \gamma) A_{c}^{\prime}(\tau+i \gamma)-\left.\mathrm{Op} p_{\partial M}\left(\bar{r}(\tau) \circ \bar{a}^{\prime}(\tau)\right)_{\gamma}\right|_{N-1}\right)
\end{aligned}
$$

(see Section 3). Here $\bar{a}$ and $\bar{r}$ are the formal symbols of the operator $A$ and its parametrix $R$ defined up to the boundary, o stands for the product of formal symbols, $A_{c}(\tau)$ and $A_{c}^{-1}(\tau)$ are the conormal symbol of $A$ and its inverse, $\operatorname{Tr}^{\prime}$ means the trace of a pseudodifferential operator on the boundary of $M$, and $N$ is the order of approximation, $N \geq \operatorname{dim} M$. The subscript $\gamma$ signalls for a formal complex shift of the real argument,

$$
a_{\gamma}(\tau)=\sum_{k=0}^{\infty} \frac{a^{(k)}(\tau)}{k !}(i \gamma h)^{k},
$$

$\gamma$ defining the weight line. 
It should be mentioned that more or less similar formulas were previously obtained in various particular cases, see e.g. [PR90] or [SSS97]. We have plucked up the courage to publish formula (0.1) because to our mind it seems new in such a form and such generality. The only assumptions we impose are that the symbols do not depend on the normal variable near the boundary (translation invariance in the terminology of [MN96a]), and that $A_{c}(\tau)$ and $A_{c}^{-1}(\tau)$ are holomorphic in a strip around the weight line.

As but one application of formula (0.1) we extend the result of Schulze, Sternin and Shatalov [SSS97] to the case of pseudodifferential operators. Namely, combining (0.1) with arguments of Section 4 ibid. thinking of $M$ as half of a compact manifold without boundary by doubling across the boundary, we arrive at a nice index formula of the Atiyah-Singer type for operators satisfying the symmetry condition of [SSS97] and possessing a meromorphic extension to the strip between the weight line and the symmetry axis $\Gamma_{0}$. More precisely,

$$
\text { ind } A=-\left(\frac{1}{2} \sum_{\substack{z \in \operatorname{spec} A_{c} \\ \Im z=0}} \operatorname{rank} z+\sum_{\substack{z \in \operatorname{spec} A_{c} \\ \Im z \in(0, \gamma)}} \operatorname{rank} z\right) \operatorname{sgn} \gamma+\int_{S^{*} M} \operatorname{AS}(A),
$$

$S^{*} M$ being the cosphere bundle of $M$ and AS $(A)$ being the Atiyah-Singer integrand manufactured from the principal interior symbol of $A$ and differential-geometric information in $M$ by local operations.

We use the machinery of cone pseudodifferential operators, referring the reader to the book of Schulze [Sch94]. The only difference is that we do not use the Mellin transform applying the change of variables by $r=e^{-t}$. Under this change the conical point becomes a 'cylindrical end' and Mellin pseudodifferential operators become Fourier pseudodifferential operators on the weight line $\Im \tau=\gamma$. Such a modification has the advantage that we do not have to switch between Mellin and Fourier representations of pseudodifferential operators. In particular, the compatibility condition for interior and conormal symbols looks much simpler for the Fourier representation.

Our next goal is to simplify formula (0.1), in particular, to reduce its interior contribution to the Atiyah-Singer integral over the cosphere bundle. Unfortunately, we have succeeded only in the case $\operatorname{dim} M=2$. The reduced formula is

$$
\text { ind } \begin{aligned}
A= & \frac{1}{4 \pi^{2}} \int_{S^{*} M} \frac{1}{6} \operatorname{tr}\left(a_{0}^{-1} \partial a_{0}\right)^{3}-\frac{1}{2} \operatorname{tr} \Omega^{0}\left(a_{0}^{-1} \partial a_{0}\right)-\frac{1}{2} \operatorname{tr} \Omega^{1}\left(\partial a_{0} a_{0}^{-1}\right) \\
& +\frac{1}{2 \pi i} \overline{\operatorname{Tr}}\left(A_{c}^{-1}(\tau+i \gamma) A_{c}^{\prime}(\tau+i \gamma)-i \gamma\left(A_{c}^{-1}(\tau+i \gamma) A_{c}^{\prime}(\tau+i \gamma)\right)^{\prime}\right) \\
& +\frac{1}{2 \pi i} \widetilde{\operatorname{Tr}}\left(a_{0}^{-1} a_{1}-\frac{i}{2} a_{0}^{-1} \frac{\partial a_{0}}{\partial \xi} a_{0}^{-1} \frac{\partial a_{0}}{\partial x}\right),
\end{aligned}
$$

$\Omega^{0}$ and $\Omega^{1}$ being the curvature forms of the bundles $E^{0}$ and $E^{1}$ and $\operatorname{tr}$ meaning the matrix trace of matrix-valued functions. 
The first summand on the right-hand side of $(0.3)$ is the usual AtiyahSinger integral. The next two summands contain the functionals $\overline{\mathrm{Tr}}$ (regularised trace) and $\widetilde{\operatorname{Tr}}$ (formal trace) introduced by Melrose [Me195]. In particular, the second summand is the 'eta' invariant (or rather one half of it) of $A_{c}(\tau)$ shifted formally to the real axis. On the other hand, the functional $\operatorname{Tr}$ is non-zero on functions $a(x, \xi, \tau)$ on $\left(T^{*} \partial M\right) \times \mathbb{R}$ positively homogeneous of degree $-1=-\operatorname{dim} \partial M$ in $(\xi, \tau)$. For such functions it takes the form

$$
\widetilde{\operatorname{Tr}} a=\left.\frac{1}{2 \pi i} \int_{T^{*} \partial M} \operatorname{tr} a(x, \xi, \tau)\right|_{\tau=-1} ^{\tau=1} d \xi d x .
$$

The advantage of formula $(0.3)$ is that it contains merely principal symbols of $A$, namely the principal interior symbol $a_{0}$ and the conormal symbol $A_{c}(\tau)$, which control the Fredholm property of $A$ acting on the Sobolev space of weight $\gamma$. The lower-order term $a_{1}(x, \xi, \tau)$ entering into the third summand of $(0.3)$ is completely determined by the conormal symbol.

Clearly, formula $(0.3)$ can be extended to symbols which are not translation invariant near the boundary.

We consider the example of the Cauchy-Riemann operator on the complex plane $\mathbb{C}$ treating the point at infinity as a conical point. This is a particular case of the Riemann-Roch theorem in the interpretation of Melrose [Me193, 6.3]. This example shows that all the three terms in (0.3) may be different from zero. It is curious enough that they are half-integer (if non-zero).

It would be interesting to extend formula (0.3) to higher dimensions, but even in the case of half-space $\mathbb{R}_{+}^{n}$ this seems to be a non-trivial homological problem.

\section{Formal symbols}

Recall an algebraic index formula on a smooth manifold, cf. [Fed74].

Let $M$ be a smooth manifold and $E^{0}, E^{1}$ vector bundles on $M$. A formal symbol $\bar{a} \in \operatorname{Symb}\left(M ; E^{0}, E^{1}\right)$ is defined by its local expressions and transitions rules. In a local chart $U \subset M$ and for given trivialisations of the bundles $E^{0}, E^{1}$ over $U$ the local expression is a formal series

$$
\begin{aligned}
\bar{a} & =\bar{a}^{U} \\
& =a_{0}(x, \xi)+h a_{1}(x, \xi)+h^{2} a_{2}(x, \xi)+\ldots,
\end{aligned}
$$

where $h$ is a formal parameter and $a_{k}(x, \xi)$ are functions on $U \times \mathbb{R}^{n}$ with values in the space of $\left(r^{1} \times r^{0}\right)$-matrices of complex numbers. Here, $n$ is the dimension of $M$ and $r^{0}, r^{1}$ stand for the ranks of $E^{0}$ and $E^{1}$ respectively. These expansions form an associative algebra with respect to the product

$$
\bar{a} \circ \bar{b}=\sum_{\alpha \in \mathbb{Z}_{+}^{n}} \frac{(-i h)^{|\alpha|}}{\alpha !} \frac{\partial^{\alpha} a}{\partial \xi^{\alpha}} \frac{\partial^{\alpha} b}{\partial x^{\alpha}}
$$


usually called a star-product or the Leibniz product.

For a change of coordinates $y=f(x)$ in $U \cap V$ we define a transition rule by

$$
\begin{aligned}
& \bar{a}^{U}(x, \xi) \\
& \quad=\left.\bar{a}^{V}\left(f(x), \xi f^{\prime-1}(x)\right) \circ \exp \left(\frac{i}{h} \xi f^{\prime-1}(x)\left(f(x)+f^{\prime}(x)(y-x)-f(y)\right)\right)\right|_{y=x},
\end{aligned}
$$

the symbol multiplication on the right-hand side being taken with respect to the variables $y$ and $\xi$ while $x$ is fixed. The vector (or rather the covector) $\eta=\xi f^{\prime-1}(x)$ in coordinates is defined by the equality

$$
\xi_{i}=\eta_{j} \frac{\partial f^{j}(x)}{\partial x^{i}}
$$

and so (1.1) is a formal version of the change of variables for pseudodifferential operators. Note that (1.1) does not contain negative powers of $h$ because of the second order zero in $y-x$ in the exponent.

Changing frames of the bundles results in the following rule

$$
\bar{a}^{U}=g_{U V}^{1} \bar{a}^{V} \circ g_{V U}^{0}
$$

For the leading term $a_{0}(x, \xi)$ we have

$$
a_{0}^{U}=g_{U V}^{1} f^{*} a_{0}^{V} g_{V U}^{0},
$$

where $\left(f^{*} a^{V}\right)(x, \xi)=a^{V}\left(f(x), \xi f^{\prime-1}(x)\right)$. So $a_{0}(x, \xi)$ varies like a function on $T^{*} M$ with values in $\operatorname{Hom}\left(\pi^{*} E^{0}, \pi^{*} E^{1}\right)$.

The higher order terms vary in a more complicated way, in particular,

$$
a_{1}^{U}=a_{1}^{V}(f(x), \eta)+\frac{i}{2} \eta_{k} \frac{\partial^{2} f^{k}}{\partial x^{i} \partial x^{j}} \frac{\partial^{2}}{\partial \xi_{i} \partial \xi_{j}} a_{0}^{V}(f(x), \eta)
$$

where $\eta=\xi f^{\prime-1}(x)$. Choosing a torsion-free connection $\nabla$ on $M$, we may express (1.3) in another way by saying that

$$
a_{1}(x, \xi)-\frac{i}{2} \xi_{k} \Gamma_{i j}^{k} \frac{\partial^{2} a_{0}(x, \xi)}{\partial \xi_{i} \partial \xi_{j}}
$$

behaves like a function on $T^{*} M$ under change of variables, $\Gamma_{i j}^{k}$ being connection coefficients. Similarly,

$$
a_{1}(x, \xi)+i \frac{\partial a_{0}(x, \xi)}{\partial \xi_{i}} \Gamma_{i}^{0}
$$

behaves like a homomorphism of bundles, $\Gamma_{i}^{0}$ being connection coefficients for the bundle $E^{0}$. 
Given a function $a(x, \xi)$ on $T^{*} M$ with values in $\operatorname{Hom}\left(\pi^{*} E^{0}, \pi^{*} E^{1}\right)$, we can always construct a formal symbol $\bar{a}$ with the leading term $a_{0}(x, \xi)=$ $a(x, \xi)$. To this end choose a covering $\left(U_{l}\right)$ by coordinate charts, local trivialisations of the bundles and a partition of unity $\left(\rho_{\iota}\right)$. For a chart $U_{\iota}$, we define the symbol $\overline{\rho_{l} a}=\rho_{\iota}(x) a(x, \xi)$ to consist of the leading term only, for chosen trivialization. Other local expressions for the symbol $\overline{\rho_{\iota} a}$ are defined by (1.1) and (1.2). Summing all the symbols $\overline{\rho_{l} a}$, we obtain the desired symbol $\bar{a}$.

Clearly, if the local functions $a_{k}^{U}(x, \xi)$ are classical symbols of order $m-k, m \in \mathbb{R}$, on $U \times \mathbb{R}$ (for example, if they are homogeneous of order $m-k$ in $\xi$ for large $|\xi|)$, then so are the functions $a_{k}^{V}$ for any other local representation. The symbols with this property we denote by $\mathrm{Symb}^{m}$.

The calculus of formal symbols is very similar to the calculus of complete symbols of classical pseudodifferential operators. The only difference is that for the formal symbols not only the asymptotic behaviour for large $|\xi|$ is relevant but the behaviour of their coefficients for small $|\xi|$ as well. For example, formal symbols vanishing for large $|\xi|$ are meaningful objects unlike complete symbols.

It is clear that for a classical complete symbol of order $m$ we can construct a formal symbol whose local coefficients $a_{k}(x, \xi)$ coincide with the homogeneous components of degree $m-k$ of the complete symbol, for large $|\xi|$. In this case we say that the corresponding pseudodifferential operator $A$ and the formal symbol $\bar{a}$ are compatible.

For a pair $(A, \bar{a}), A$ and $\bar{a}$ being compatible, we define a regularisation of $A$ as follows. Let

$$
\left.\bar{a}\right|_{N}=\sum_{k=0}^{N-1} a_{k}(x, \xi)
$$

be a partial sum for $\bar{a}$ with $h=1$. Let further

$$
\mathrm{Op}\left(\left.\bar{a}\right|_{N}\right):=\sum_{l} \mathrm{Op}\left(\left.\rho_{\iota} \bar{a}^{U_{\iota}}\right|_{N}\right) \tilde{\rho}_{t}
$$

be the pseudodifferential operator on $M$ defined by a coordinate covering $\left(U_{l}\right)$, partition of unity $\left(\rho_{l}\right)$ and trivialisations of the bundles. The functions $\tilde{\rho}_{\iota}$ are required to be identically equal to 1 on the supports of $\rho_{\iota}$ but still satisfy supp $\tilde{\rho}_{\iota} \subset U_{\iota}$. (In this way we obtain what will be referred to as 'covering functions'.) As usual, Op means a standard pseudodifferential operator on $\mathbb{R}^{n}$ defined by the symbol $\left.\rho_{l} \bar{a}\right|_{N}$. Of course, the operator (1.4) depends on the choices mentioned above. The operator

$$
\left.\operatorname{reg}\right|_{N} A:=A-O p\left(\left.\bar{a}\right|_{N}\right)
$$

is of order $m-N$ and thus is of trace class in each Sobolev space $H^{s}(M)$ provided $N-m>n$ (we suppose $M$ is compact).

Define a regularised trace of $A$ by

$$
\left.\operatorname{Tr} \operatorname{reg}\right|_{N} A:=\operatorname{Tr}\left(A-\operatorname{Op}\left(\left.\bar{a}\right|_{N}\right)\right),
$$


$A$ and $\bar{a}$ being compatible. The properties of the regularised trace are described in the following proposition.

Proposition 1.1 Let $A$ be a classical pseudodifferential operator of order $m$ and let $\bar{a} \in \mathrm{Symb}^{m}$ be a formal symbol compatible with A. Then:

1) the regularised trace $\operatorname{Tr}\left(A-\mathrm{Op}\left(\left.\bar{a}\right|_{N}\right)\right)$ is independent of the choices of coordinate charts, trivialisations and partition of unity.

2) the regularised trace vanishes on commutators, more precisely,

$$
\left.\operatorname{Tr} \operatorname{reg}\right|_{N} A B=\left.\operatorname{Tr} \operatorname{reg}\right|_{N} B A
$$

if the regularisations of $A B$ and $B A$ are defined by the formal symbols $\bar{a} \circ \bar{b}$ and $\bar{b} \circ \bar{a}$, respectively.

The proofs may be found in [Fed74].

If $\bar{a} \in \mathrm{Symb}^{m}$ with $m<-n$, then the operator $\mathrm{Op}\left(\left.\bar{a}\right|_{N}\right)$ belongs to the trace class and its trace is correctly defined by the symbol $\bar{a}$ and the truncation number $N$. In this case we abbreviate the notation of its trace to

$$
\left.\operatorname{Tr} \bar{a}\right|_{N}:=\operatorname{Tr} \mathrm{Op}\left(\left.\bar{a}\right|_{N}\right) .
$$

This number may be interpreted as an integral over $T^{*} M$, more precisely,

$$
\left.\operatorname{Tr} \bar{a}\right|_{N}=\frac{1}{(2 \pi)^{n}} \int_{M}\left(\left.\int_{\mathbb{R}^{n}} \operatorname{tr} \bar{a}\right|_{N} d \xi\right) d x
$$

where the inner integral defines a density on $M$ not depending on the local representation of the formal symbol $\bar{a}$. In (1.5) and subsequently, the notation tr stands for the matrix trace of the coefficients $a_{k}(x, \xi)$.

We are interested in the application of the notion of formal symbol to the index theorem for elliptic operators. If $A$ is an elliptic pseudodifferential operator then there exists a parametrix $R$ such that $1-R A$ and $1-A R$ are smoothing operators. If further, $\bar{a}$ and $\bar{r}$ are formal symbols compatible with the operators $A$ and $R$ the following algebraic index formula holds

$$
\text { ind } A=\left.\operatorname{Tr}(1-\bar{r} \circ \bar{a})\right|_{N}-\left.\operatorname{Tr}(1-\bar{a} \circ \bar{r})\right|_{N}
$$

for any $N \geq n$. This formula is an intermediate step between the analytical index formula

$$
\text { ind } A=\operatorname{Tr}(1-R A)-\operatorname{Tr}(1-A R)
$$

and the topological index formula due to Atiyah and Singer.

For further references let us write down the Atiyah-Singer index formula for two-dimensional manifolds,

$$
\text { ind } A=\frac{1}{4 \pi^{2}} \int_{S^{*} M} \frac{1}{6} \operatorname{tr}\left(a^{-1} \partial a\right)^{3}-\frac{1}{2} \operatorname{tr} \Omega^{0}\left(a^{-1} \partial a\right)-\frac{1}{2} \operatorname{tr} \Omega^{1}\left(\partial a a^{-1}\right) \text {, }
$$


where $S^{*} M$ is the cosphere bundle oriented as a boundary of the coball bundle $B^{*} M$ with the orientation form

$$
\begin{aligned}
\frac{\omega^{n}}{n !} & =\frac{\left(d \xi_{i} \wedge d x^{i}\right)^{n}}{n !} \\
& =d \xi_{1} \wedge d x^{1} \wedge \ldots \wedge d \xi_{n} \wedge d x^{n},
\end{aligned}
$$

the covariant differential $\partial$ of homomorphisms $a: E^{0} \rightarrow E^{1}$ is defined on sections $u \in C^{\infty}\left(M, E^{0}\right)$ by

$$
(\partial a) u=\partial^{1}(a u)-a \partial^{0} u,
$$

$\partial^{0}, \partial^{1}$ being connections on $E^{0}, E^{1}$, and $\Omega^{0}, \Omega^{1}$ are the curvature forms of $E^{0}, E^{1}$.

We finish this section by a remark that the calculus of formal symbols is a particular case of a more general calculus, called deformation quantisation, on an arbitrary symplectic manifold. Here, the symplectic manifold in question is $T^{*} M$.

\section{Pseudodifferential operators}

From the point of view of analysis, a manifold with a conical point is in fact a smooth manifold with boundary $(M, \partial M)$. A collar neighborhood of the boundary is diffeomorphic to a cylinder $\partial M \times I$, where $I$ is the interval $0 \leq r<1$.

Typical differential operators considered in the cone theory (see e.g. Schulze [Sch94]) are of the form

$$
A=r^{-p} \sum_{k=0}^{m} a_{k}(r)\left(-r \frac{\partial}{\partial r}\right)^{k}
$$

close to the boundary, with coefficients $a_{k}(r)$ smooth up to $r=0$, their values being differential operators on $\partial M$ of order $m-k$. Usually one considers the weight factor in front of the sum with $p=m$ but this is not necessary, we allow any $p \in \mathbb{R}$. The analysis of such operators is based on the Mellin transform which evokes a technical difficulty caused by switching between Fourier and Mellin representations of pseudodifferential operators. To avoid it we pass from the collar neighbourhood to a cylindrical end by changing the variable $r=e^{-t}, 0<t<\infty$. The operator (2.1) becomes

$$
e^{p t} \sum_{k=0}^{m} a_{k}\left(e^{-t}\right)\left(\frac{\partial}{\partial t}\right)^{k} .
$$

The exponential stabilisation of coefficients as $t \rightarrow \infty$ is inherited from the smoothness up to $r=0$ in (2.1). The exponential weight factor $e^{p t}$ may be included into basis sections of the bundle $E^{1}$ in the cylindrical charts. 
This means that we pass from the frame $e_{1}\left(x^{\prime}, t\right), \ldots, e_{r^{1}}\left(x^{\prime}, t\right), r^{1}$ being the rank of $E^{1}$, to the frame

$$
e^{\prime}{ }_{1}\left(x^{\prime}, t\right)=e_{1}\left(x^{\prime}, t\right) e^{p t}, \ldots, e_{r^{1}}^{\prime}\left(x^{\prime}, t\right)=e_{r^{1}}\left(x^{\prime}, t\right) e^{p t}
$$

In this new basis the operator (2.2) becomes

$$
\sum_{k=0}^{m} a_{k}\left(e^{-t}\right)\left(\frac{\partial}{\partial t}\right)^{k} .
$$

In the sequel by a conical point we mean a cylindrical end $\mathbb{R}_{+} \times \partial M$ and exponential stabilisation of all the objects in question at $t=+\infty$. We assume that near the end everything has a cylindrical structure. So, vector bundles over the end are lifted from the boundary $\partial M$, local charts have the form $U_{\iota}=\mathbb{R}_{+} \times U^{\prime}{ }_{\iota}$ with $U^{\prime}{ }_{\iota} \subset \partial M$, thus resulting in the splitting of coordinates $x=\left(t, x^{\prime}\right), x^{\prime} \in U^{\prime}{ }_{\iota}$. Next, for a cylindrical chart $U_{i}$, we have

$$
\begin{aligned}
& \rho_{\iota}(x)=\rho_{\infty}(t) \rho_{t}^{\prime}\left(x^{\prime}\right), \\
& \tilde{\rho}_{l}(x)=\tilde{\rho}_{\infty}(t) \tilde{\rho}_{t}^{\prime}\left(x^{\prime}\right),
\end{aligned}
$$

where $\left(\rho_{l}\right)$ is a partition of unity and $\left(\tilde{\rho}_{l}\right)$ the set of covering functions. We emphasise that $\rho_{\infty} \in C^{\infty}\left(\mathbb{R}_{+}\right)$is equal to 1 close to $t=+\infty$ and vanishes close to $t=0$.

Introduce the weighted Sobolev spaces $H^{s, \gamma}(M, E)$ by requiring $\rho_{\iota} u \in$ $H^{s}\left(\mathbb{R}^{n}\right)$ for the interior charts and $e^{\gamma t} \rho_{\iota} u \in H^{s}\left(\mathbb{R}^{n}\right)$ for the cylindrical charts, the norm being equal to

$$
\|u\|_{s, \gamma}^{2}=\sum_{l}\left\|\rho_{\iota} u\right\|_{s}^{2}+\sum_{j}\left\|e^{\gamma t} \rho_{j} u\right\|_{s}^{2}
$$

where $\iota$ runs over interior charts and $j$ over cylindrical charts. For a $C^{\infty}$ function $u\left(t, x^{\prime}\right)$ of compact support in $\mathbb{R}_{+} \times \mathbb{R}^{n-1}$, the weighted Sobolev norm $\left\|e^{\gamma t} u\right\|_{s}$ may be written as

$$
\int_{\mathbb{R}^{n-1}} \int_{\Gamma_{\gamma}}\left(1+|\Re \tau|^{2}+\left|\xi^{\prime}\right|^{2}\right)^{2 s}\left|\hat{u}\left(\tau, \xi^{\prime}\right)\right|^{2} d \tau d \xi^{\prime}
$$

where $\Gamma_{\gamma}=\{\tau \in \mathbb{C}: \Im \tau=\gamma\}$ is a horizontal line in the complex plane to be referred to as the weight line, and $\hat{u}\left(\tau, \xi^{\prime}\right)$ means the Fourier transform of $u\left(t, x^{\prime}\right)$ which is an entire function in $\tau$ rapidly decreasing on horizontal lines.

There is an embedding

$$
H^{s_{1}, \gamma_{1}} \hookrightarrow H^{s_{2}, \gamma_{2}}
$$

for $s_{1} \geq s_{2}, \gamma_{1} \geq \gamma_{2}$, which is compact for $s_{1}>s_{2}, \gamma_{1}>\gamma_{2}$ (but not for $\left.s_{1}>s_{2}, \gamma_{1}=\gamma_{2}\right)$, Hilbert-Schmidt for $s_{1}>s_{2}+n / 2, \gamma_{1}>\gamma_{2}$, and of trace 
class for $s_{1}>s_{2}+n, \gamma_{1}>\gamma_{2}$ (see e.g. [FS96]). The space $H^{-s,-\gamma}$ is dual for $H^{s, \gamma}$ with respect to the usual paring given in local coordinates by

$$
(u, v)=\int_{\mathbb{R}^{n}} u \bar{v} d x, \quad \text { for } \quad u \in H^{s, \gamma}, v \in H^{-s,-\gamma} .
$$

We next describe pseudodifferential operators acting between weighted Sobolev spaces. We confine ourselves to operators which in the cylindrical charts are independent of $t$ apart from a weight factor $e^{p t}$ which we always omit including it into the frame of the bundle $E^{1}$ as was explained before. For the purposes of index theory this class is sufficient.

The cone pseudodifferential operators are of the form

$$
A u=\rho_{0} A_{\text {int }} \tilde{\rho}_{0} u+\rho_{\infty} A_{\gamma} \tilde{\rho}_{\infty} u
$$

where $\rho_{\infty} \in C^{\infty}\left(\mathbb{R}_{+}\right)$is a non-negative function satisfying $\rho_{\infty} \equiv 0$ near $t=0$ and $\rho_{\infty} \equiv 1$ near $t=+\infty$, and $\rho_{0}=1-\rho_{\infty}$. The operator $A_{\text {int }}$ is a classical pseudodifferential operator of order $m \in \mathbb{R}$ in the interior of $M$. The operator $A_{\gamma}$ is defined by a so-called conormal symbol $A_{c}(\tau)$, $\tau \in \Gamma_{\gamma}$, which is a parametre-dependent pseudodifferential operator of order $m$ on $\partial M$. We assume that $A_{c}(\tau)$ has an analytic extension to a strip $|\Im \tau-\gamma|<\varepsilon$ around the weight line and on each horizontal line in this strip is a parametre-dependent pseudodifferential operator on $\partial M$ uniformly with respect to $\Im \tau$ in a smaller strip $|\Im \tau-\gamma| \leq \varepsilon_{1}$, with $\varepsilon_{1}<\varepsilon$. Then, $A_{\gamma}$ acts on functions $u(t) \in C_{\text {comp }}^{\infty}\left(\mathbb{R}_{+}, C^{\infty}(\partial M, E)\right)$ as

$$
\begin{aligned}
A_{\gamma} u(t) & =\mathrm{Op}_{t}^{\gamma}\left(A_{c}(\tau)\right) u(t) \\
& :=\frac{1}{2 \pi} \int_{\Gamma_{\gamma}} e^{i t \tau} A_{c}(\tau) \hat{u}(\tau) d \tau \\
& =e^{-\gamma t} \mathrm{Op}_{t}\left(A_{c}(\tau+i \gamma)\right) e^{\gamma t} u(t) .
\end{aligned}
$$

Here we denote by $\mathrm{Op}_{t}^{\gamma}$ a standard pseudodifferential operator with the shifted integration line $\varsigma \tau=\gamma$ while the notation $\mathrm{Op}_{t}=\mathrm{Op}_{t}^{0}$ means that the integration line is the real axis. In both cases the symbols $A_{c}$ are operator-valued functions.

It is well known, cf. Schulze [Sch94], that such operators are bounded, when mapping from $H^{s, \gamma}$ to $H^{s-m, \gamma}$.

The operators $A_{\text {int }}$ and $A_{\gamma}$ in the cone theory are not independent. They satisfy a very important compatibility condition expressing the fact that $A_{\gamma}$ arises in practice as a result of redefinition of $A_{\mathrm{int}}$. Roughly speaking, it means that, for any $\rho_{1}, \rho_{2} \in C_{\text {comp }}^{\infty}\left(\mathbb{R}_{+}\right)$, the difference

$$
\rho_{1} A_{\text {int }} \rho_{2}-\rho_{1} A_{\gamma} \rho_{2}
$$

is a smoothing operator on $M$ and thus belongs to the trace class in the spaces $H^{s, \gamma}$. More precisely this condition can be formulated in terms of formal symbols. 
Let $\bar{a}$ be a formal symbol of $A_{\text {int }}$. We assume that in the cylindrical charts it takes the form

$$
\begin{aligned}
\bar{a}\left(t, \tau, x^{\prime}, \xi^{\prime}\right) & \equiv \bar{a}\left(\tau, x^{\prime}, \xi^{\prime}\right) \\
& \in \operatorname{Symb}^{m}\left(M ; E^{0}, E^{1}\right) .
\end{aligned}
$$

Being independent of $t$, the symbol $\bar{a}$ may be considered as a formal symbol on $\partial M$ and we can construct a parametre-dependent operator $\mathrm{Op}_{\partial M}\left(\left.\bar{a}(\tau)\right|_{N}\right)$ as described in Section 1. Define a formal imaginary shift of the parametre $\tau$ by

$$
\begin{aligned}
\bar{a}_{\gamma}(\tau) & =\bar{a}(\tau+i h \gamma) \\
& :=\sum_{k=0}^{\infty} h^{k} \frac{1}{k !} \frac{\partial^{k}}{\partial \tau^{k}} \bar{a}(\tau)(i \gamma)^{k} \\
& =e^{\gamma t} \bar{a}(\tau) \circ e^{-\gamma t} .
\end{aligned}
$$

The compatibility condition in a more precise form means that, for any $N$, the difference

$$
R_{N}(\tau)=A_{c}(\tau+i \gamma)-\mathrm{Op}{ }_{\partial M}\left(\left.\bar{a}(\tau+i h \gamma)\right|_{N}\right), \quad \tau \in \mathbb{R}
$$

is a classical parametre-dependent pseudodifferential operator of order $m-$ $N$ on $\partial M$ bearing the following trace norm estimate, for $m-N<-n+1$,

$$
\left\|\frac{\partial^{k}}{\partial \tau^{k}} R_{N}(\tau)\right\|_{1} \leq C\langle\tau\rangle^{m-N-k+n-1}
$$

where $\|\cdot\|_{1}$ stands for the trace norm of the operator in any space $H^{s}(\partial M)$. For standard pseudodifferential operators on $\mathbb{R}^{n-1}$ whose symbols are homogeneous in $\left(\tau, \xi^{\prime}\right)$ outside a ball these estimates are valid but in general we have to postulate them.

Operator (2.5) may be written as

$$
\rho_{1}\left(A_{\text {int }}-\mathrm{Op}\left(\left.\bar{a}\right|_{N}\right)\right) \rho_{2}-\rho_{1} e^{-\gamma t} \mathrm{Op}_{t}\left(R_{N}(\tau)\right) e^{\gamma t} \rho_{2},
$$

so, for compactly supported $\rho_{1}$ and $\rho_{2}$, both summands are trace class operators in $H^{s, \gamma}$, for each $\gamma$.

Denoting by $\mathcal{C}^{m, \gamma}$ the class of such operators (obeying the compatibility condition), we obtain what is called the cone algebra. Indeed, one can verify that the composition of $A \in \mathcal{C}^{m, \gamma}$ and $B \in \mathcal{C}^{l, \gamma}$ gives an operator $B A \in \mathcal{C}^{l+m, \gamma}$. In the case when the order $m$ coincides with the exponent $p$ of the weight factor $e^{p t}$ in (2.2), this algebra was studied by the second author (see e.g. [Sch94] and references therein). It is worth pointing out that the cone theory deals with Mellin pseudodifferential operators near a conical point, but the results may be easily transported to our case by the change of variables $r=e^{-t}$. 


\section{Algebraic index theorem}

In this section we consider elliptic operators of the type (2.3). Recall that ellipticity means that the principal homogeneous symbol $a_{0}(x, \xi)$ is invertible for $\xi \neq 0$ (interior ellipticity) and that the conormal symbol $A_{c}(\tau)$ is an invertible pseudodifferential operator on $\partial M$ for any $\tau \in \Gamma_{\gamma}$ (conormal ellipticity).

The interior ellipticity implies that there exists a formal symbol $\bar{r}$ on $M$ such that $1-\bar{r} \circ \bar{a}$ and $1-\bar{a} \circ \bar{r}$ vanish for $|\xi|$ large enough. We denote by $R_{\text {int }}$ a pseudodifferential operator on $M$ compatible with $\bar{r}$ (see Section 1).

The conormal ellipticity allows one to define an operator

$$
R_{\gamma}=\mathrm{Op} p_{t}^{\gamma}\left(A_{c}^{-1}(\tau)\right)
$$

The operators $1-R_{\text {int }} A_{\text {int }}$ and $1-A_{\text {int }} R_{\text {int }}$ are smoothing in the interior of $M$ (that is, being multiplied by a cut-off function $\rho$ of compact support away from the boundary of $M$ ), hence the compatibility condition for $R_{\text {int }}$ and $R_{\gamma}$ follows from (2.5). Indeed,

$$
R_{\gamma}-R_{\mathrm{int}}=R_{\gamma}\left(1-A_{\mathrm{int}} R_{\mathrm{int}}\right)+R_{\gamma}\left(A_{\mathrm{int}}-A_{\gamma}\right) R_{\mathrm{int}}
$$

and we see that both summands are smoothing in the interior.

We introduce an operator

$$
\begin{aligned}
R & =\rho_{0} R_{\text {int }} \tilde{\rho}_{0}+\rho_{\infty} R_{\gamma} \tilde{\rho}_{\infty} \\
& \in \mathcal{C}^{-m, \gamma}
\end{aligned}
$$

called a parametrix of $A$.

Theorem 3.1 The operators

$$
\begin{gathered}
1-R A: \quad H^{s, \gamma} \rightarrow H^{s, \gamma}, \\
1-A R: \quad H^{s-m, \gamma} \rightarrow H^{s-m, \gamma}
\end{gathered}
$$

are of trace class and the following index formula holds

$$
\text { ind } \begin{aligned}
A= & \left.\operatorname{Tr} \rho_{0}(1-\bar{r} \circ \bar{a})\right|_{N}-\left.\operatorname{Tr} \rho_{0}(1-\bar{a} \circ \bar{r})\right|_{N} \\
& +\left.\frac{1}{2 \pi i} \int_{-\infty}^{\infty} \operatorname{Tr}^{\prime} \operatorname{reg}\right|_{N-1} A_{c}^{-1}(\tau+i \gamma) A_{c}^{\prime}(\tau+i \gamma) d \tau,
\end{aligned}
$$

for any $N \geq n$.

Let us explain the notations and give some comments. First of all, $\mathrm{Tr}^{\prime}$ denotes a trace on $\partial M$ and reg $\left.\right|_{N-1}$ denotes a regularisation of the corresponding $A_{c}^{-1} A_{c}^{\prime}$ (here the prime means the derivative with respect to $\tau$ ) by means of its formal symbol as was explained in Section 1. It is important for this formal symbol (and thus the regularisation) to be defined by the 
interior formal symbols $\bar{a}$ and $\bar{r}$ as follows. In a cylindrical chart the symbols $\bar{a}=\bar{a}(\tau)$ and $\bar{r}=\bar{r}(\tau)$, being independent of $t$, may be considered as formal symbols on $\partial M$ depending on the parametre $\tau \in \mathbb{R}$. Denote by

$$
\begin{aligned}
& \bar{a}_{\gamma}(\tau)=\bar{a}(\tau+i h \gamma), \\
& \bar{r}_{\gamma}(\tau)=\bar{r}(\tau+i h \gamma)
\end{aligned}
$$

the formally shifted symbols defined by (2.6). Then we must take the symbol

$$
\bar{r}_{\gamma}(\tau) \circ \bar{a}_{\gamma}^{\prime}(\tau)=\left(\bar{r}(\tau) \circ \bar{a}^{\prime}(\tau)\right)_{\gamma}
$$

to regularise $A_{c}^{-1} A_{c}^{\prime}$. In other words,

$$
\begin{aligned}
& \left.\operatorname{reg}\right|_{N-1} A_{c}^{-1}(\tau+i \gamma) A_{c}^{\prime}(\tau+i \gamma) \\
& \quad=A_{c}^{-1}(\tau+i \gamma) A_{c}^{\prime}(\tau+i \gamma)-\mathrm{Op} \mathrm{p}_{\partial M}\left(\left.\left(\bar{r}(\tau) \circ \bar{a}^{\prime}(\tau)\right)_{\gamma}\right|_{N-1}\right)
\end{aligned}
$$

The cut-off function $\rho_{0}$ entering into the first two terms in (3.2) is inessential. Indeed, for two different choices of $\rho_{0}(t)$, we have $\Delta \rho_{0} \in$ $C_{\text {comp }}^{\infty}\left(\mathbb{R}_{+}\right)$, so the difference of the corresponding expressions is

$$
\frac{1}{2 \pi} \int_{0}^{\infty} \Delta \rho_{0}(t) d t \int_{-\infty}^{\infty} d \tau\left(\left.\operatorname{Tr}^{\prime}(1-\bar{r}(\tau) \circ \bar{a}(\tau))\right|_{N}-\left.\operatorname{Tr}^{\prime}(1-\bar{a}(\tau) \circ \bar{r}(\tau))\right|_{N}\right) .
$$

But the expression in the parentheses vanishes because it is equal to the index of $A_{c}(\tau)$ which is zero for $A_{c}(\tau)$ is invertible.

Proof. The starting point is formula (1.7). Using (2.3) and (3.1), we obtain

$$
\begin{aligned}
1-R A= & 1-\rho_{0} R_{\mathrm{int}} \tilde{\rho}_{0} \rho_{0} A_{\mathrm{int}} \tilde{\rho}_{0}-\rho_{0} R_{\mathrm{int}} \tilde{\rho}_{0} \rho_{\infty} A_{\gamma} \tilde{\rho}_{\infty} \\
& -\rho_{\infty} R_{\gamma} \tilde{\rho}_{\infty} \rho_{0} A_{\mathrm{int}} \tilde{\rho}_{0}-\rho_{\infty} R_{\gamma} \tilde{\rho}_{\infty} \rho_{\infty} A_{\gamma} \tilde{\rho}_{\infty}
\end{aligned}
$$

and

$$
\begin{aligned}
1-A R= & 1-\rho_{0} A_{\text {int }} \tilde{\rho}_{0} \rho_{0} R_{\text {int }} \tilde{\rho}_{0}-\rho_{\infty} A_{\gamma} \tilde{\rho}_{\infty} \rho_{0} R_{\text {int }} \tilde{\rho}_{0} \\
& -\rho_{0} A_{\text {int }} \tilde{\rho}_{0} \rho_{\infty} R_{\gamma} \tilde{\rho}_{\infty}-\rho_{\infty} A_{\gamma} \tilde{\rho}_{\infty} \rho_{\infty} R_{\gamma} \tilde{\rho}_{\infty}
\end{aligned}
$$

The corresponding terms in (3.4) and (3.5) differ by the order of factors. We will transform these terms pairwise and write $A B \sim C, B A \sim D$ if $A B-C$ and $B A-D$ are of trace class and their traces coincide. For example,

$$
A B \sim A^{\prime} B^{\prime}, \quad B A \sim B^{\prime} A^{\prime}
$$

if both $A-A^{\prime}$ and $B-B^{\prime}$ are of trace class since

$$
\begin{aligned}
& \operatorname{Tr}\left(A B-A^{\prime} B^{\prime}\right)=\operatorname{Tr}\left(A-A^{\prime}\right) B+\operatorname{Tr} A^{\prime}\left(B-B^{\prime}\right), \\
& \operatorname{Tr}\left(B A-B^{\prime} A^{\prime}\right)=\operatorname{Tr} B\left(A-A^{\prime}\right)+\operatorname{Tr}\left(B-B^{\prime}\right) A^{\prime} .
\end{aligned}
$$

As

$$
\mathrm{Op}\left(\left.\rho_{0} \bar{r} \circ \tilde{\rho}_{0} \circ \rho_{0} \bar{a} \circ \tilde{\rho}_{0}\right|_{N}\right) \equiv \mathrm{Op}\left(\left.\rho_{0} \bar{r} \circ \rho_{0} \bar{a}\right|_{N}\right),
$$


the first pair in (3.4) and (3.5) may be replaced by another one equivalent to it, namely

$$
\mathrm{Op}\left(\left.\rho_{0} \bar{r} \circ \rho_{0} \bar{a}\right|_{N}\right), \quad \mathrm{Op}\left(\left.\rho_{0} \bar{a} \circ \rho_{0} \bar{r}\right|_{N}\right),
$$

which is clear from Proposition 1.1.

To transform the next pair we use the pseudolocality of pseudodifferential operators. Since $\rho_{0} \rho_{\infty}$ has a compact support in $\mathbb{R}_{+}$, we may replace $\rho_{0}$ by $\rho_{1}$ and $\rho_{\infty}$ by $\rho_{2}$, each of them having a compact support in $\mathbb{R}_{+}$, such that $\rho_{0} \rho_{\infty}=\rho_{1} \rho_{2}$. By $\tilde{\rho}_{1}, \tilde{\rho}_{2}$ we denote "covering" functions for $\rho_{1}, \rho_{2}$ still being of compact support in $\mathbb{R}_{+}$.

Let us justify, for example, the replacement of $\rho_{\infty}$ by $\rho_{2}$. We choose $\rho_{2}$ so that $\left(\rho_{\infty}-\rho_{2}\right) \tilde{\rho}_{0} \equiv 0$. Then

$$
\left(\rho_{\infty}-\rho_{2}\right) A_{\gamma} \tilde{\rho}_{\infty} \rho_{0} R_{\text {int }} \tilde{\rho}_{0}
$$

is a trace class operator by the pseudolocality property since $\rho_{\infty}-\rho_{2}$ and $\rho_{0}$ have disjoint supports (details may be found in [FS96]). The trace of this operator is 0 since

$$
\begin{aligned}
\operatorname{Tr}\left(\rho_{\infty}-\rho_{2}\right) O \tilde{\rho}_{0} & =\operatorname{Tr}\left(\rho_{\infty}-\rho_{2}\right) \rho_{\infty} O \tilde{\rho}_{0} \\
& =\operatorname{Tr} \rho_{\infty} O \tilde{\rho}_{0}\left(\rho_{\infty}-\rho_{2}\right) \\
& =0 .
\end{aligned}
$$

The corresponding term with the order interchanged is identically zero.

Similarly we may replace step by step $\rho_{0}$ by $\rho_{1}, \tilde{\rho}_{0}$ by $\tilde{\rho}_{1}$ and $\tilde{\rho}_{\infty}$ by $\tilde{\rho}_{2}$. As a result we obtain an equivalent pair of the form

$$
\rho_{1} R_{\text {int }} \tilde{\rho}_{1} \rho_{2} A_{\gamma} \tilde{\rho}_{2}, \quad \rho_{2} A_{\gamma} \tilde{\rho}_{2} \rho_{1} R_{\text {int }} \tilde{\rho}_{1} .
$$

Now, using (3.6) and compatibility condition (2.5), we replace $\rho_{2} A_{\gamma} \tilde{\rho}_{2}$ by $\rho_{2} A_{\text {int }} \tilde{\rho}_{2}$ obtaining the pair

$$
\rho_{1} R_{\text {int }} \tilde{\rho}_{1} \rho_{2} A_{\text {int }} \tilde{\rho}_{2}, \quad \rho_{2} A_{\text {int }} \tilde{\rho}_{2} \rho_{1} R_{\text {int }} \tilde{\rho}_{1} .
$$

Since

$$
\mathrm{Op}\left(\left.\rho_{1} \bar{r} \circ \rho_{2} \bar{a}\right|_{N}\right) \equiv \mathrm{Op}\left(\left.\rho_{0} \bar{r} \circ \rho_{\infty} \bar{a}\right|_{N}\right),
$$

we may make use of Proposition 1.1 once again to replace the latter pair by

$$
\mathrm{Op}\left(\left.\rho_{0} \bar{r} \circ \rho_{\infty} \bar{a}\right|_{N}\right), \quad \mathrm{Op}\left(\left.\rho_{\infty} \bar{a} \circ \rho_{0} \bar{r}\right|_{N}\right) .
$$

Similarly, the third pair in (3.4), (3.5) may be replaced by

$$
\mathrm{Op}\left(\left.\rho_{\infty} \bar{r} \circ \rho_{0} \bar{a}\right|_{N}\right), \quad \mathrm{Op}\left(\left.\rho_{0} \bar{a} \circ \rho_{\infty} \bar{r}\right|_{N}\right) .
$$

In the last pair the operators may be treated as pseudodifferential operators on the half-axis $t \in \mathbb{R}_{+}$with operator-valued symbols. The cut-offs $\tilde{\rho}_{\infty}$ may be omitted by pseudolocality. Denote temporarily

$$
\begin{aligned}
& O(t, \tau)=\rho_{\infty}(t) A_{c}(\tau), \\
& P(t, \tau)=\rho_{\infty}(t) A_{c}^{-1}(\tau) .
\end{aligned}
$$


In these notations the last pair in $(3.4),(3.5)$ is

$$
\mathrm{Op}_{t}^{\gamma}(P(t, \tau)) \mathrm{Op}_{t}^{\gamma}(O(t, \tau)), \quad \mathrm{Op}_{t}^{\gamma}(O(t, \tau)) \mathrm{Op}_{t}^{\gamma}(P(t, \tau)) .
$$

It is equivalent to the pair

$$
\mathrm{Op}_{t}^{\gamma}\left(\left.P(t, \tau) \circ O(t, \tau)\right|_{N}\right), \quad \mathrm{Op}_{t}^{\gamma}\left(\left.O(t, \tau) \circ P(t, \tau)\right|_{N}\right),
$$

which is due to the theorem on the regularised trace of a product from [FS96] similar to Proposition 1.1, n. 2). Here o means the symbol product of operator-valued symbols on the shifted real axis and the subscript $N$ means truncation, that is

$$
\left.P \circ O\right|_{N}=\sum_{k=0}^{N-1} \frac{(-i)^{k}}{k !} \frac{\partial^{k} P}{\partial \tau^{k}} \frac{\partial^{k} O}{\partial t^{k}}
$$

Gathering all the terms (3.7)-(3.10), we obtain

$$
\begin{aligned}
1-R A \sim & 1-\mathrm{Op}\left(\left.\rho_{0} \bar{r} \circ \rho_{0} \bar{a}\right|_{N}\right)-\mathrm{Op}\left(\left.\rho_{0} \bar{r} \circ \rho_{\infty} \bar{a}\right|_{N}\right) \\
& -\mathrm{Op}\left(\left.\rho_{\infty} \bar{r} \circ \rho_{0} \bar{a}\right|_{N}\right)-\mathrm{Op} \gamma\left(\left.\rho_{\infty} A_{c}^{-1}(\tau) \circ \rho_{\infty} A_{c}(\tau)\right|_{N}\right), \\
1-A R \sim & 1-\mathrm{Op}\left(\left.\rho_{0} \bar{a} \circ \rho_{0} \bar{r}\right|_{N}\right)-\mathrm{Op}\left(\left.\rho_{\infty} \bar{a} \circ \rho_{0} \bar{r}\right|_{N}\right) \\
& -\mathrm{Op}\left(\left.\rho_{0} \bar{a} \circ \rho_{\infty} \bar{r}\right|_{N}\right)-\mathrm{Op} \gamma\left(\left.\rho_{\infty} A_{c}(\tau) \circ \rho_{\infty} A_{c}^{-1}(\tau)\right|_{N}\right) .
\end{aligned}
$$

Further,

$$
\begin{aligned}
\left.\rho_{\infty} A_{c}^{-1}(\tau) \circ \rho_{\infty} A_{c}(\tau)\right|_{N} & =\left.\rho_{\infty} A_{c}^{-1}(\tau) \circ\left(1-\rho_{0}\right) A_{c}(\tau)\right|_{N} \\
& =\rho_{\infty}-\left.\rho_{\infty} A_{c}^{-1}(\tau) \circ \rho_{0} A_{c}(\tau)\right|_{N}
\end{aligned}
$$

and similarly

$$
\left.\rho_{\infty} A_{c}(\tau) \circ \rho_{\infty} A_{c}^{-1}(\tau)\right|_{N}=\rho_{\infty}-\left.\rho_{\infty} A_{c}(\tau) \circ \rho_{0} A_{c}^{-1}(\tau)\right|_{N}
$$

This results in

$$
\begin{aligned}
1-R A \sim & \mathrm{Op}\left(\left.\rho_{0}(1-\bar{r} \circ \bar{a})\right|_{N}\right) \\
& +\operatorname{Op}_{t}^{\gamma}\left(\left.\rho_{\infty} A_{c}^{-1}(\tau) \circ \rho_{0} A_{c}(\tau)\right|_{N}\right)-\mathrm{Op}\left(\left.\rho_{\infty} \bar{r} \circ \rho_{0} \bar{a}\right|_{N}\right), \\
1-A R \sim & \mathrm{Op}\left(\left.\rho_{0}(1-\bar{a} \circ \bar{r})\right|_{N}\right) \\
& +\mathrm{Opp}_{t}^{\gamma}\left(\left.\rho_{\infty} A_{c}(\tau) \circ \rho_{0} A_{c}^{-1}(\tau)\right|_{N}\right)-\mathrm{Op}\left(\left.\rho_{\infty} \bar{a} \circ \rho_{0} \bar{r}\right|_{N}\right) .
\end{aligned}
$$

The first terms on the right-hand sides of (3.11) and (3.12) are trace class operators and the difference of their traces gives precisely the interior terms in (3.2). In the rest terms all the symbols have compact supports in $t \in \mathbb{R}_{+}$since $\rho_{\infty} \rho_{0}$ has, so we may regard them as operators on the cylinder $\mathbb{R}_{+} \times \partial M$.

To feel free with the exponential weight functions let us formulate the following lemma. 
Lemma 3.2 Let $a(t, \tau)$ be an operator-valued symbol with a compact support on $\mathbb{R}_{+}$. Then, for $N$ large enough, the operator

$$
e^{p t} \mathrm{Op}_{t}^{\gamma}(a(t, \tau)) e^{-p t}-\mathrm{Op}_{t}^{\gamma}\left(\left.e^{p t} a(t, \tau) \circ e^{-p t}\right|_{N}\right)
$$

is of trace class and its trace vanishes.

Proof. This is a particular case of the theorem on the regularised trace of a product. We take

$$
\begin{aligned}
& A=O \mathrm{pp}_{t}^{\gamma}\left(e^{p t} a(t, \tau)\right) \\
& B=\mathrm{Op}_{t}^{\gamma}\left(e^{-p t}\right) .
\end{aligned}
$$

Note that $e^{-p t}$ may be replaced by any function coinciding with $e^{-p t}$ on the support of $a$, which is due to pseudolocality.

Consider the last two terms on the right in (3.11). We will use the notations

$$
\tilde{A}(\tau)=A(\tau+i \gamma), \quad \tau \in \mathbb{R},
$$

for the complex shift and

$$
\bar{a}_{\gamma}\left(t, \tau, x^{\prime}, \xi^{\prime}\right)=\bar{a}\left(t, \tau+i h \gamma, x^{\prime}, \xi^{\prime}\right)
$$

for the formal complex shift. By (2.4),

$$
\mathrm{Op} p_{t}^{\gamma}\left(\left.\rho_{\infty} A_{c}^{-1} \circ \rho_{0} A_{c}\right|_{N}\right)=e^{-\gamma t} \mathrm{Op} p_{t}\left(\left.\rho_{\infty} \tilde{A}_{c}^{-1} \circ \rho_{0} \tilde{A}_{c}\right|_{N}\right) e^{\gamma t}
$$

and

$$
e^{\gamma t} \mathrm{Op}\left(\left.\rho_{\infty} \bar{r} \circ \rho_{0} \bar{a}\right|_{N}\right) e^{-\gamma t} \sim \mathrm{Op}\left(\left.\rho_{\infty} \bar{r}_{\gamma} \circ \rho_{0} \bar{a}_{\gamma}\right|_{N}\right),
$$

the last equality being a consequence of Lemma 3.2 and (2.6). Further, using the equality $\mathrm{Op}=\mathrm{Op}_{t} \mathrm{Op} \mathrm{p}_{\partial M}$, we obtain finally for the last two terms in (3.11) the equivalent expression

$$
\mathrm{Op}_{t}\left(\left.\rho_{\infty} \tilde{A}_{c}^{-1} \circ \rho_{0} \tilde{A}_{c}\right|_{N}-\mathrm{Op} \mathrm{p}_{\partial M}\left(\left.\rho_{\infty} \bar{r}_{\gamma}(\tau) \circ \rho_{0} \bar{a}_{\gamma}(\tau)\right|_{N}\right)\right)
$$

and similarly for the last two terms in (3.12)

$$
\mathrm{Op}_{t}\left(\left.\rho_{\infty} \tilde{A}_{c} \circ \rho_{0} \tilde{A}_{c}^{-1}\right|_{N}-\mathrm{Op}{ }_{\partial M}\left(\left.\rho_{\infty} \bar{a}_{\gamma}(\tau) \circ \rho_{0} \bar{r}_{\gamma}(\tau)\right|_{N}\right)\right) .
$$

The operators $(3.13),(3.14)$ are of trace class due to the compatibility condition, so the conjugations by $e^{-\gamma t}$ do not affect their traces.

The next transformation goes as follows. We carry the factor $\rho_{0}$ through $\tilde{A}_{c}$ and $\bar{a}_{\gamma}$ in (3.13), thus obtaining

$$
\begin{aligned}
\mathrm{Op}_{t} & \left\{\mathrm{Op}_{\partial M}\left(\left.\rho_{\infty}\left(1-\bar{r}_{\gamma} \circ \bar{a}_{\gamma}\right) \circ \rho_{0}\right|_{N}\right)\right. \\
& \left.-\left.\rho_{\infty} \tilde{A}_{c}^{-1} \circ\left[\tilde{A}_{c}, \rho_{0}\right]\right|_{N}+\mathrm{Op} p_{\partial M}\left(\left.\rho_{\infty} \bar{r}_{\gamma} \circ\left[\bar{a}_{\gamma}, \rho_{0}\right]\right|_{N}\right)\right\} .
\end{aligned}
$$


In (3.14) we move $\rho_{\infty}$ through $\tilde{A}_{c}$ and $\bar{a}_{\gamma}$ as follows

$$
\begin{aligned}
\rho_{\infty} \tilde{A}_{c} & =\tilde{A}_{c} \rho_{\infty}-\left[\tilde{A}_{c}, \rho_{\infty}\right] \\
& =\tilde{A}_{c} \rho_{\infty}+\left[\tilde{A}_{c}, \rho_{0}\right] .
\end{aligned}
$$

This gives

$$
\begin{aligned}
\mathrm{Op}_{t} & \left\{\left.\tilde{A}_{c} \circ \rho_{\infty} \circ \rho_{0} \tilde{A}_{c}^{-1}\right|_{N}-\mathrm{Op} \mathrm{p}_{\partial M}\left(\left.\bar{a}_{\gamma}(\tau) \circ \rho_{\infty} \circ \rho_{0} \bar{r}_{\gamma}(\tau)\right|_{N}\right)\right. \\
& \left.+\left.\left[\tilde{A}_{c}, \rho_{0}\right] \circ \rho_{0} \tilde{A}_{c}^{-1}\right|_{N}-\mathrm{Op} \operatorname{p}_{M}\left(\left.\left[\bar{a}_{\gamma}(\tau), \rho_{0}\right] \circ \rho_{0} \bar{r}_{\gamma}(\tau)\right|_{N}\right)\right\} .
\end{aligned}
$$

Now, expressions $(3.15),(3.16)$ are trace class operators in $H^{s, 0}$. Moreover, expressions in the curly brackets in (3.15), (3.16) are trace class operators on $\partial M$ since each operator-valued symbol is regularised by subtracting a non-trace class part. We may change the order of operators in (3.16) changing simultaneously the order of symbols, thus arriving at

$$
\begin{aligned}
\mathrm{Op}_{t} & \left\{\mathrm{Op} \operatorname{p}_{\partial M}\left(\left.\rho_{0}\left(1-\bar{r}_{\gamma} \circ \bar{a}_{\gamma}\right) \circ \rho_{\infty}\right|_{N}\right)\right. \\
& \left.+\left.\rho_{0} \tilde{A}_{c}^{-1} \circ\left[\tilde{A}_{c}, \rho_{0}\right]\right|_{N}-\mathrm{Op} \operatorname{p}_{2 M}\left(\left.\rho_{0} \bar{r}_{\gamma}(\tau) \circ\left[\bar{a}_{\gamma}(\tau), \rho_{0}\right]\right|_{N}\right)\right\} .
\end{aligned}
$$

Finally, taking traces of (3.15) and (3.17) and subtracting yields

$$
-\frac{1}{2 \pi} \int_{0}^{\infty} d t \int_{-\infty}^{\infty} d \tau \operatorname{Tr}^{\prime}\left(\left.\tilde{A}_{c}^{-1} \circ\left[\tilde{A}_{c}, \rho_{0}\right]\right|_{N}-\mathrm{Op} \partial M\left(\left.\bar{r}_{\gamma}(\tau) \circ\left[\bar{a}_{\gamma}(\tau), \rho_{0}\right]\right|_{N}\right)\right) .
$$

Now,

$$
\begin{aligned}
{\left[\tilde{A}_{c}, \rho_{0}\right] } & =\sum_{k=1}^{\infty} \frac{(-i)^{k}}{k !} \frac{d^{k} \tilde{A}_{c}}{d \tau^{k}} \frac{d^{k} \rho_{0}}{d t^{k}} \\
{\left[\bar{a}_{\gamma}(\tau), \rho_{0}\right] } & =\sum_{k=1}^{\infty} \frac{(-i)^{k}}{k !} \frac{\partial^{k} \bar{a}_{\gamma}(\tau)}{\partial \tau^{k}} \frac{d^{k} \rho_{0}}{d t^{k}} .
\end{aligned}
$$

Integration over $t$ yields zero unless $k=1$. In the latter case

$$
\int_{0}^{\infty} \frac{d \rho_{0}}{d t} d t=-1
$$

and we obtain the desired result.

Remark 3.3 If a symbol $a(\tau)$ decreases sufficiently fast then formal complex shifts do not affect the value of the integral, that is

$$
\int_{-\infty}^{\infty} a(\tau) d \tau=\left.\int_{-\infty}^{\infty} a(\tau+i h \gamma)\right|_{N-1} d \tau
$$

Indeed, the terms of $a(t+i h \gamma)$ have the form

$$
\frac{(i h \gamma)^{k}}{k !} \frac{d^{k}}{d \tau^{k}} a(\tau)
$$


so the integration gives zero, for $k$ large enough. This allows us to replace expression (3.3) by the formally shifted one. When shifting by $-i h \gamma$, we obtain another expression for the integrand in (3.2) which is sometimes more convenient,

$$
\text { ind } \begin{aligned}
A= & \left.\operatorname{Tr} \rho_{0}(1-\bar{r} \circ \bar{a})\right|_{N}-\left.\operatorname{Tr} \rho_{0}(1-\bar{a} \circ \bar{r})\right|_{N} \\
& +\frac{1}{2 \pi i} \int_{-\infty}^{\infty} \operatorname{Tr}^{\prime}\left(\left.A_{c}^{-1}(\tau+i \gamma-i h \gamma) A_{c}^{\prime}(\tau+i \gamma-i h \gamma)\right|_{N-1}\right. \\
& \left.-O p_{\partial M}\left(\left.\bar{r}(\tau) \circ \bar{a}^{\prime}(\tau)\right|_{N-1}\right)\right) d \tau .
\end{aligned}
$$

Remark 3.4 A special case of (3.18) arises when $A_{c}^{-1}(\tau) A_{c}^{\prime}(\tau)$ is a meromorphic operator-valued function with finite-dimensional Laurent coefficients at poles. In this case the integral in (3.18) may be reduced to the integral over the real axis (if there are no real poles) and a finite sum of residues in the poles between the real axis and the weight line. This is known as the Relative Index Theorem (see e.g. Melrose and Mendoza [MM83]). Indeed, for a meromorphic function $a(z)$ with a regular behaviour as $\Re z \rightarrow \infty$, we have

$$
\frac{1}{2 \pi i} \int_{-\infty}^{\infty}\left(a(\tau+i \gamma)-\left.a(\tau+i h \gamma)\right|_{N}\right) d \tau=-\operatorname{sgn} \gamma \sum_{\varsigma z \in(0, \gamma)} \operatorname{res} a(z),
$$

so that (3.18) becomes

$$
\text { ind } \begin{aligned}
A= & \left.\operatorname{Tr} \rho_{0}(1-\bar{r} \circ \bar{a})\right|_{N}-\left.\operatorname{Tr} \rho_{0}(1-\bar{a} \circ \bar{r})\right|_{N} \\
& +\frac{1}{2 \pi i} \int_{-\infty}^{\infty} \operatorname{Tr}^{\prime}\left(A_{c}^{-1}(\tau) A_{c}^{\prime}(\tau)-O \operatorname{p}_{\partial M}\left(\left.\bar{r}(\tau) \circ \bar{a}^{\prime}(\tau)\right|_{N-1}\right)\right) d \tau \\
& -\operatorname{sgn} \gamma \sum_{s z \in(0, \gamma)} \operatorname{Tr}^{\prime} \operatorname{res} A_{c}^{-1}(z) A_{c}^{\prime}(z) .
\end{aligned}
$$

Some corrections should be made when some poles meet the real axis (see an example in Section 5).

Remark 3.5 There exists another regularisation of $A_{c}^{-1} A_{c}^{\prime}$ leading to the so-called 'eta' invariant. The latter was introduced by Melrose [Mel95] as a generalisation of the Atiyah-Patodi-Singer $\eta$-invariant. If $A(\tau)$ is a parameter-dependent pseudodifferential operator on the boundary $\partial M$, we denote by $\overline{\operatorname{Tr}} A(\tau)$ the following number. For $N$ large enough, the derivative $A^{(N)}(\tau)$ is of trace class and $\operatorname{Tr}^{\prime} A^{(N)}(\tau)$ has an asymptotic expansion in negative powers of $|\tau|$ as $\tau \rightarrow \pm \infty$. Hence it follows that

$$
I_{N}(T)=\int_{-T}^{T} d \tau_{N} \int_{0}^{\tau_{N}} d \tau_{N-1} \ldots \int_{0}^{\tau_{1}} \operatorname{Tr}^{\prime} A^{(N)}(\tau) d \tau
$$


also has an asymptotic expansion in negative powers of $T$ up to a summand $p_{1}(T)+p_{2}(T) \log T$, where $p_{1}$ and $p_{2}$ are polynomials. Then $\overline{\operatorname{Tr}} A(\tau)$ is, by definition, the constant term in the asymptotic expansion of (3.20). Clearly,

$$
\overline{\operatorname{Tr}} A(\tau)=\int_{-\infty}^{\infty} \operatorname{Tr}^{\prime} A(\tau) d \tau
$$

for each trace class operator $A(\tau)$ with sufficiently fast decreasing trace norm. In these notations formula (3.18) becomes

$$
\text { ind } \begin{aligned}
A= & \left.\operatorname{Tr} \rho_{0}(1-\bar{r} \circ \bar{a})\right|_{N}-\left.\operatorname{Tr} \rho_{0}(1-\bar{a} \circ \bar{r})\right|_{N} \\
& +\left.\frac{1}{2 \pi i} \overline{\operatorname{Tr}} A_{c}^{-1}(\tau+i \gamma-i h \gamma) \circ A_{c}^{\prime}(\tau+i \gamma-i h \gamma)\right|_{N-1} \\
& -\frac{1}{2 \pi i} \overline{\operatorname{Tr}} \operatorname{Op}_{\partial M}\left(\left.\bar{r}(\tau) \circ \bar{a}^{\prime}(\tau)\right|_{N-1}\right) .
\end{aligned}
$$

The second term on the right-hand side of this formula is usually denoted by $\frac{1}{2} \eta(A)$ and $\eta(A)$ is called the $\eta$-invariant.

\section{Two-dimensional case}

For the two-dimensional case formula (3.21) admits further simplifications. We use here direct tiresome computations which can not be generalised to higher dimensions. Nevertheless the result seems to be of interest for it shows the structure of the index formula in terms of leading symbols (interior and conormal). Besides the Atiyah-Singer integral (1.8) and the $\eta$ invariant our formula contains an additional term non-vanishing in general. It has a structure very similar to the Wodzicki non-commutative residue.

Theorem 4.1 If $\operatorname{dim} M=2$, then formula (0.3) holds.

To work on a manifold, we need an invariant symbolic calculus. In general such a calculus is given by deformation quantization. Fortunately, for $n=2$, we need a few first terms, so the full calculus is not needed.

Any symbol may be described locally by means of its coefficients

$$
a_{0}, a_{1}, \ldots
$$

where only $a_{0}$ behaves like a function, or globally by means of its principal symbol $a_{0}$ and subprincipal symbol ${ }^{1}$

$$
\tilde{a}_{1}=a_{1}+i \frac{\partial a_{0}}{\partial \xi_{i}} \Gamma_{i}^{0}-\frac{i}{2} \frac{\partial^{2} a_{0}}{\partial \xi_{i} \partial \xi_{j}} \Gamma_{i j}^{k} \xi_{k}
$$

\footnotetext{
${ }^{1}$ Not to be confused with the common notion of subprincipal symbol in partial differential equations.
} 
which also behaves like a function (see Section 1). It is easy to verify that if

$$
\bar{a} \circ \bar{b}=a_{0} b_{0}+h\left(-i \frac{\partial a_{0}}{\partial \xi_{i}} \frac{\partial b_{0}}{\partial x^{i}}+a_{0} b_{1}+a_{1} b_{0}\right)+\ldots,
$$

then for the subprincipal symbol we have the following rule

$$
\widetilde{(\bar{a} \circ \bar{b}})_{1}=-i \frac{\partial a_{0}}{\partial \xi_{i}} \nabla_{i} b_{0}+a_{0} \tilde{b}_{1}+\tilde{a}_{1} b_{0}
$$

where

$$
\nabla_{i} b_{0}=\frac{\partial b_{0}}{\partial x^{i}}+\frac{\partial b_{0}}{\partial \xi_{j}} \Gamma_{j i}^{k} \xi_{k}+\Gamma_{i}^{1} b_{0}-b_{0} \Gamma_{i}^{0}
$$

is the so-called covariant derivative.

Under changes of variables $\nabla_{i} b_{0}$ behave like coordinates of a covector. We introduce also

$$
\nabla b_{0}=d \xi_{i} \frac{\partial b_{0}}{\partial \xi_{i}}+d x^{i} \nabla_{i} b_{0},
$$

the so-called covariant differential. It is a function on $T^{*} M$ with values in $\operatorname{Hom}\left(E^{0}, E^{1}\right) \otimes \Lambda^{1}$.

We will not go further into invariant calculus combining the local approach with the invariant one. So, we start with the local expression

$$
\frac{1}{4 \pi^{2}}\left(\left.\operatorname{tr}(1-\bar{r} \circ \bar{a})\right|_{2}-\left.\operatorname{tr}(1-\bar{a} \circ \bar{r})\right|_{2}\right) \frac{\omega^{2}}{2} .
$$

As we know, it does not depend on the coordinate system giving a density on $M$. We have

$$
\begin{aligned}
& \left.(1-\bar{r} \circ \bar{a})\right|_{2}=1-r_{0} a_{0}+\left(i \frac{\partial r_{0}}{\partial \xi_{i}} \frac{\partial a_{0}}{\partial x^{i}}-r_{0} a_{1}-r_{1} a_{0}\right) \\
& +\left(\frac{1}{2} \frac{\partial^{2} r_{0}}{\partial \xi_{i} \partial \xi_{j}} \frac{\partial^{2} a_{0}}{\partial x^{i} \partial x^{j}}+i \frac{\partial r_{0}}{\partial \xi_{i}} \frac{\partial a_{1}}{\partial x^{i}}+i \frac{\partial r_{1}}{\partial \xi_{i}} \frac{\partial a_{0}}{\partial x^{i}}-r_{0} a_{2}-r_{1} a_{1}-r_{2} a_{0}\right)
\end{aligned}
$$

and a similar expression for $\left.(1-\bar{a} \circ \bar{r})\right|_{2}$. Taking the difference of traces, we see that the terms not containing derivatives cancel, whence

$$
\begin{aligned}
Q:= & \left.\operatorname{tr}(1-\bar{r} \circ \bar{a})\right|_{2}-\left.\operatorname{tr}(1-\bar{a} \circ \bar{r})\right|_{2} \\
= & i \operatorname{tr}\left(\frac{\partial r_{0}}{\partial \xi_{i}} \frac{\partial a_{0}}{\partial x^{i}}-\frac{\partial r_{0}}{\partial x^{i}} \frac{\partial a_{0}}{\partial \xi_{i}}\right) \\
& +i \operatorname{tr}\left(\frac{\partial r_{0}}{\partial \xi_{i}} \frac{\partial a_{1}}{\partial x^{i}}-\frac{\partial r_{0}}{\partial x^{i}} \frac{\partial a_{1}}{\partial \xi_{i}}\right)+i \operatorname{tr}\left(\frac{\partial r_{1}}{\partial \xi_{i}} \frac{\partial a_{0}}{\partial x^{i}}-\frac{\partial r_{1}}{\partial x^{i}} \frac{\partial a_{0}}{\partial \xi_{i}}\right) \\
& +\frac{1}{2} \operatorname{tr}\left(\frac{\partial^{2} r_{0}}{\partial \xi_{i} \partial \xi_{j}} \frac{\partial^{2} a_{0}}{\partial x^{i} \partial x^{j}}-\frac{\partial^{2} r_{0}}{\partial x^{i} \partial x^{j}} \frac{\partial^{2} a_{0}}{\partial \xi_{i} \partial \xi_{j}}\right) .
\end{aligned}
$$

The first term may be rewritten as

$$
i \frac{\partial}{\partial \xi_{i}} \operatorname{tr} r_{0} \frac{\partial a_{0}}{\partial x^{i}}-i \frac{\partial}{\partial x^{i}} \operatorname{tr} r_{0} \frac{\partial a_{0}}{\partial \xi_{i}} \text {. }
$$


Being multiplied by $\omega^{2} / 2$ it gives

$$
i d\left(\operatorname{tr}\left(r_{0} d a_{0}\right) \wedge \omega\right)=-i d\left(\operatorname{tr}\left(d r_{0} a_{0}\right) \wedge \omega\right) .
$$

Similarly may be transformed the second and the third terms on the right in (4.2). The last term may be rewritten as

$\frac{1}{2} \frac{\partial}{\partial \xi_{i}} \operatorname{tr}\left(\frac{\partial r_{0}}{\partial x^{j}} \frac{\partial^{2} a_{0}}{\partial \xi_{j} \partial x^{i}}-\frac{\partial^{2} r_{0}}{\partial \xi_{j} \partial x^{i}} \frac{\partial a_{0}}{\partial x^{j}}\right)-\frac{1}{2} \frac{\partial}{\partial x^{i}} \operatorname{tr}\left(\frac{\partial r_{0}}{\partial x^{j}} \frac{\partial^{2} a_{0}}{\partial \xi_{j} \partial x_{i}}-\frac{\partial^{2} r_{0}}{\partial \xi_{i} \partial \xi_{j}} \frac{\partial a_{0}}{\partial x^{j}}\right)$.

Multiplying by $\omega^{2} / 2$, we get

$$
\frac{1}{2} d\left(\operatorname{tr}\left(\frac{\partial r_{0}}{\partial x^{j}} d\left(\frac{\partial a_{0}}{\partial \xi_{j}}\right)-d\left(\frac{\partial r_{0}}{\partial \xi_{j}}\right) \frac{\partial a_{0}}{\partial x^{j}}\right) \wedge \omega\right)
$$

Thus,

$$
Q \frac{\omega^{2}}{2}=d(f \wedge \omega)
$$

where

$$
f=i \operatorname{tr} r_{0} d a_{0}+i \operatorname{tr}\left(r_{1} d a_{0}-d r_{0} a_{1}\right)+\frac{1}{2} \operatorname{tr}\left(\frac{\partial r_{0}}{\partial x^{j}} d\left(\frac{\partial a_{0}}{\partial \xi_{j}}\right)-d\left(\frac{\partial r_{0}}{\partial \xi_{j}}\right) \frac{\partial a_{0}}{\partial x^{j}}\right) .
$$

Now we would like to switch to invariant objects in (4.3). As for the exterior differential $d$ in front, it is globally defined provided $f$ is an oneform. But this is not the case, and we have first to examine the contributions of non-invariant terms.

The first term $i \operatorname{tr} r_{0} d a_{0}$ is invariant. The second one may be written in the form

$$
\begin{aligned}
& i \operatorname{tr}\left(\tilde{r}_{1} d a_{0}-d r_{0} \tilde{a}_{1}\right) \\
& \quad-\frac{1}{2} \operatorname{tr}\left(\frac{\partial^{2} r_{0}}{\partial \xi_{i} \partial \xi_{j}} \Gamma_{i j}^{k} \xi_{k} d a_{0}-d r_{0} \frac{\partial^{2} a_{0}}{\partial \xi_{i} \partial \xi_{j}} \Gamma_{i j}^{k} \xi_{k}\right) \\
& \quad+\operatorname{tr}\left(\frac{\partial r_{0}}{\partial \xi_{i}} \Gamma_{i}^{1} d a_{0}-d r_{0} \frac{\partial a_{0}}{\partial \xi_{i}} \Gamma_{i}^{0}\right)
\end{aligned}
$$

where the first summand is invariant. Finally, the last term in (4.3) becomes globally defined if we replace the derivatives in $x^{i}$ and differentials by covariant ones,

$$
\frac{1}{2} \operatorname{tr}\left(\nabla_{j} r_{0} \nabla\left(\frac{\partial a_{0}}{\partial \xi_{j}}\right)-\nabla\left(\frac{\partial r_{0}}{\partial \xi_{j}}\right) \nabla_{j} a_{0}\right)
$$

Applying $\nabla$ to $\partial a_{0} / \partial \xi_{j}$, we take into account that $\partial a_{0} / \partial \xi_{j}$ behaves like a vector, and so $\nabla$ means a covariant differential of the vector field.

Examining the contributions of the non-invariant terms at a point $x_{0} \in$ $M$, we introduce geodesic coordinates centered at $x_{0}$ and the frames in $E^{0}$, 
$E^{1}$ parallel along the rays since the result is independent of coordinates. In these coordinates all the $\Gamma_{i j}^{k}, \Gamma_{i}^{0}$ and $\Gamma_{i}^{1}$ vanish at $x_{0}$ and their derivatives define curvatures:

$$
\begin{aligned}
& \frac{\partial \Gamma_{i j}^{k}}{\partial x^{l}}=-\frac{1}{6}\left(R_{i j l}^{k}+R_{j i l}^{k}\right), \\
& \left.\frac{\partial \Gamma_{i}^{0}}{\partial x^{j}}=-\frac{1}{2} \Omega_{i j}^{0}\left(x_{0}\right)\right), \\
& \left.\frac{\partial \Gamma_{i}^{1}}{\partial x^{j}}=-\frac{1}{2} \Omega_{i j}^{1}\left(x_{0}\right)\right) .
\end{aligned}
$$

Since $\Gamma_{i j}^{k}\left(x_{0}\right)=0$, the contribution of the second term in (4.4) is equal (up to a constant factor) to

$$
\begin{aligned}
\operatorname{tr} & \left(\frac{\partial^{2} r_{0}}{\partial \xi_{i} \partial \xi_{j}} \xi_{k} R_{i j l}^{k} d x^{l} \wedge d a_{0}+d r_{0} \wedge \frac{\partial^{2} a_{0}}{\partial \xi_{l} \partial \xi_{j}} \xi_{k} R_{i j l}^{k} d x^{l}\right) \wedge \omega \\
& =\operatorname{tr}\left(-\frac{\partial^{2} r_{0}}{\partial \xi_{i} \partial \xi_{j}} \frac{\partial a_{0}}{\partial \xi_{l}} \xi_{k} R_{i j l}^{k}+\frac{\partial r_{0}}{\partial \xi_{l}} \frac{\partial^{2} a_{0}}{\partial \xi_{i} \partial \xi_{j}} \xi_{k} R_{i j l}^{k}\right) \frac{\omega^{2}}{2} \\
& =-\operatorname{tr} \frac{\partial}{\partial \xi_{i}}\left(\frac{\partial r_{0}}{\partial \xi_{j}} \frac{\partial a_{0}}{\partial \xi_{l}}\right) \xi_{k} R_{i j l}^{k} \frac{\omega^{2}}{2} \\
& =-\frac{\partial}{\partial \xi_{i}} \operatorname{tr}\left(\frac{\partial r_{0}}{\partial \xi_{j}} \frac{\partial a_{0}}{\partial \xi_{l}} \xi_{k} R_{i j l}^{k}\right) \frac{\omega^{2}}{2}+\operatorname{tr} \frac{\partial r_{0}}{\partial \xi_{j}} \frac{\partial a_{0}}{\partial \xi_{l}} R_{i j l}^{i} \frac{\omega^{2}}{2}
\end{aligned}
$$

the last summand being zero since the convolution $R_{i j l}^{i}$ vanishes. On the other hand, the first summand does not contribute either because for large $\xi$ we have $r_{0}=a_{0}^{-1}$ and thus

$$
\begin{aligned}
\operatorname{tr} \frac{\partial r_{0}}{\partial \xi_{j}} \frac{\partial a_{0}}{\partial \xi_{l}} R_{i j l}^{k} & =-\operatorname{tr} a_{0}^{-1} \frac{\partial a_{0}}{\partial \xi_{j}} a_{0}^{-1} \frac{\partial a_{0}}{\partial \xi_{l}} R_{i j l}^{k} \\
& =0
\end{aligned}
$$

the last equality being due to the fact that $R_{i j l}^{k}$ is antisymmetric in $j, l$. Hence the first summand gives 0 after integration in $\xi$, for it is a complete derivative of a function with compact support.

Now the contribution of the third term in (4.4) is

$$
\begin{array}{r}
-\frac{1}{2} \operatorname{tr}\left(\frac{\partial r_{0}}{\partial \xi_{i}} \Omega_{i j}^{1} d x^{j} \wedge d a_{0}+d r_{0} \wedge \frac{\partial a_{0}}{\partial \xi_{i}} \Omega_{i j}^{0} d x^{j}\right) \wedge \omega \\
=-\frac{1}{2} \operatorname{tr} d r_{0} \wedge \Omega^{1} \wedge d a_{0}-\frac{1}{2} \operatorname{tr} d r_{0} \wedge d a_{0} \wedge \Omega^{0}
\end{array}
$$

Here we may replace $d$ by $\nabla$ since they differ by terms containing $d x^{i}$. So, the final contribution is

$$
-\frac{1}{2} d \operatorname{tr}\left(r_{0} \Omega^{1} \wedge \nabla a_{0}+r_{0} \nabla a_{0} \wedge \Omega^{0}\right)
$$


We next show that the replacement $\partial / \partial x^{j}$ by $\nabla_{j}$ in the last term in (4.3) gives no contribution. For example, consider a term

$$
d \operatorname{tr}\left(\nabla_{j} r_{0}-\frac{\partial}{\partial x^{j}} r_{0}\right) d\left(\frac{\partial a_{0}}{\partial \xi_{j}}\right) \wedge \omega
$$

The difference $\nabla_{j}-\partial / \partial x^{j}$ necessarily contains connection coefficients $\Gamma_{i}^{0}$, $\Gamma_{i}^{0}$ and $\Gamma_{i j}^{k}$. Let us take the term with $\Gamma_{i}^{1}$, that is

$$
d \operatorname{tr} \Gamma_{j}^{1} r_{0} d\left(\frac{\partial a_{0}}{\partial \xi_{j}}\right) \wedge \omega=-\frac{1}{2} \operatorname{tr} \Omega_{j k}^{1} d x^{k} r_{0} \wedge d\left(\frac{\partial a_{0}}{\partial \xi_{j}}\right) \wedge \omega
$$

in the center $x_{0}$ of a special coordinate system. But

$$
d x^{k} \wedge d\left(\frac{\partial a_{0}}{\partial \xi_{j}}\right) \wedge \omega=d x^{k} \wedge \frac{\partial^{2} a_{0}}{\partial \xi_{j} \partial \xi_{k}} d \xi^{k} \wedge \omega
$$

for $j \neq k$, and this gives zero since $\Omega_{j k}^{1}$ is antisymmetric. Precisely the same reason goes for the other terms. Moreover, it shows that $d$ and $\nabla$ may also be replaced by each other.

The final expression of (4.3) in the invariant form is

$$
\begin{aligned}
Q \frac{\omega^{2}}{2}=d & \left\{i \operatorname{tr} r_{0} \nabla a_{0} \wedge \omega\right. \\
& +i \operatorname{tr}\left(\tilde{r}_{1} \nabla a_{0}-\nabla r_{0} \tilde{a}_{1}\right) \wedge \omega-\frac{1}{2} \operatorname{tr}\left(r_{0} \Omega^{1} \wedge \nabla a_{0}+r_{0} \nabla a_{0} \wedge \Omega^{0}\right) \\
& \left.+\frac{1}{2} \operatorname{tr}\left(\nabla_{j} r_{0} \nabla \frac{\partial a_{0}}{\partial \xi_{j}}-\nabla \frac{\partial r_{0}}{\partial \xi_{j}} \nabla_{j} a_{0}\right) \wedge \omega\right\} .
\end{aligned}
$$

Having disposed of these preliminary steps, we can now return to the inner term in the index formula which is

$$
\frac{1}{4 \pi^{2}} \int_{B^{*} M} Q \frac{\omega^{2}}{2}
$$

where $B^{*} M$ is the coball bundle $|\xi| \leq 1$. The boundary of $B^{*} M$ consists of two parts, namely $S^{*} M$ and $B^{*} M$ restricted to $\partial M$. The application of the Stokes formula gives two summands.

First, we consider the integral over $S^{*} M$ and show that it is precisely the Atiyah-Singer integral (1.8). Indeed, we have $r_{0}=a_{0}^{1}$ on $S^{*} M$. Using (4.1) for $\bar{a}$ and $\bar{r}$ and equating it to 0 , we obtain

$$
i \frac{\partial a_{0}}{\partial \xi_{i}} a_{0}^{-1}\left(\nabla_{i} a_{0}\right) a_{0}^{-1}+a_{0} \tilde{r}_{1}+\tilde{a}_{1} a_{0}^{-1}=0,
$$

whence

$$
\tilde{r}_{1}=-a_{0}^{-1} \tilde{a}_{1} a_{0}^{-1}-i a_{0}^{-1} \frac{\partial a_{0}}{\partial \xi_{i}} a_{0}^{-1}\left(\nabla_{i} a_{0}\right) a_{0}^{-1} .
$$


Substitution this into (4.5) shows that the subprincipal symbol $\tilde{a}_{1}$ vanishes and the remaining terms are

$$
\begin{aligned}
i \operatorname{tr} a_{0}^{-1} \nabla a_{0} \wedge \omega+\operatorname{tr} a_{0}^{-1} \frac{\partial a_{0}}{\partial \xi_{j}} a_{0}^{-1}\left(\nabla_{j} a_{0}\right) a_{0}^{-1} \nabla a_{0} \wedge \omega \\
\quad-\frac{1}{2} \operatorname{tr}\left(a_{0}^{-1} \Omega^{1} \wedge \nabla a_{0}+a_{0}^{-1} \nabla a_{0} \wedge \Omega^{0}\right) \\
\quad+\frac{1}{2} \operatorname{tr}\left(\nabla_{j} a_{0}^{-1} \nabla \frac{\partial a_{0}}{\partial \xi_{j}}-\nabla \frac{\partial a_{0}^{-1}}{\partial \xi_{j}} \nabla_{j} a_{0}\right) \wedge \omega .
\end{aligned}
$$

Now

$$
\nabla \frac{\partial a_{0}^{-1}}{\partial \xi_{j}}=a_{0}^{-1} \nabla a_{0} a_{0}^{-1} \frac{\partial a_{0}}{\partial \xi_{j}} a_{0}^{-1}-a_{0}^{-1} \nabla\left(\frac{\partial a_{0}}{\partial \xi_{j}}\right) a_{0}^{-1}+a_{0}^{-1} \frac{\partial a_{0}}{\partial \xi_{j}} a_{0}^{-1} \nabla a_{0} a_{0}^{-1}
$$

which leads to the cancellation of the terms with second order derivatives in

$$
\begin{aligned}
\operatorname{tr} a_{0}^{-1} & \frac{\partial a_{0}}{\partial \xi_{j}} a_{0}^{-1}\left(\nabla_{j} a_{0}\right) a_{0}^{-1} \nabla a_{0} \wedge \omega \\
& +\frac{1}{2} \operatorname{tr}\left(\nabla_{j} a_{0}^{-1} \nabla \frac{\partial a_{0}}{\partial \xi_{j}}-\nabla \frac{\partial a_{0}^{-1}}{\partial \xi_{j}} \nabla_{j} a_{0}\right) \wedge \omega \\
= & \frac{1}{2} \operatorname{tr}\left(a_{0}^{-1} \frac{\partial a_{0}}{\partial \xi_{j}} a_{0}^{-1} \nabla_{j} a_{0} a_{0}^{-1} \nabla a_{0}-a_{0}^{-1} \frac{\partial a_{0}}{\partial \xi_{j}} a_{0}^{-1} \nabla a_{0} a_{0}^{-1} \nabla_{j} a_{0}\right) \wedge \omega \\
= & \frac{1}{6} \operatorname{tr}\left(a_{0}^{-1} \nabla a_{0}\right)^{3} .
\end{aligned}
$$

It remains to observe that

$$
i \operatorname{tr} a_{0}^{-1} \nabla a_{0} \wedge \omega=i d\left(\operatorname{tr}\left(a_{0}^{-1} \nabla a_{0}\right) \xi_{j} d x^{j}\right),
$$

so it contributes to a boundary term under integration. Thus, the integral over $S^{*} M$ is (we suppress the "wedge' product)

$$
\frac{1}{4 \pi^{2}} \int_{S^{*} M}-\frac{1}{2} \operatorname{tr}\left(a_{0}^{-1} \Omega^{1} \nabla a_{0}+a_{0}^{-1} \nabla a_{0} \Omega^{0}\right)+\frac{1}{6} \operatorname{tr}\left(a_{0}^{-1} \nabla a_{0}\right)^{3}
$$

which is equal (up to a sign) to the Atiyah-Singer integral.

The boundary $\partial M$ for $n=2$ is a circle, so all the bundles and connections are trivial. Hence, the coefficients $a_{0}, a_{1}, \ldots$ of the formal symbol $\bar{a}$ are functions on $\left.T^{*} M\right|_{\partial M}$ and their covariant derivatives coincide with the usual ones. We use global coordinates $x, \xi, \tau$, where $x \in \mathbb{R}(\bmod 2 \pi)$ and $\xi, \tau \in \mathbb{R}$. The result of our computation may be written in the form

$$
\begin{aligned}
\operatorname{Tr} \rho_{0} & \left.(1-\bar{r} \circ \bar{a})\right|_{2}-\left.\operatorname{Tr} \rho_{0}(1-\bar{a} \circ \bar{r})\right|_{2}=\int_{S^{*} M} \operatorname{AS}(A) \\
& -\frac{1}{4 \pi^{2}} \int_{\left.B^{*} M\right|_{\partial M}} i \operatorname{tr} r_{0} d a_{0} \wedge \omega+i \operatorname{tr}\left(r_{1} d a_{0}-d r_{0} a_{1}\right) \wedge \omega \\
& +\frac{1}{2} \operatorname{tr}\left(\frac{\partial r_{0}}{\partial x} \frac{\partial}{\partial \xi}\left(d a_{0}\right)-d\left(\frac{\partial r_{0}}{\partial \xi}\right) \frac{\partial a_{0}}{\partial x}\right) \wedge \omega+i d\left(\operatorname{tr}\left(r_{0} d a_{0}\right) \xi d x\right) .
\end{aligned}
$$


The integrand in the second integral is zero for large $r=\sqrt{\xi^{2}+\tau^{2}}$. Indeed, for $r>1$ we have $r_{0}=a_{0}^{-1}$ and, as we have seen, the integrand coincides with the Atiyah-Singer form which is now a constant multiple of $\operatorname{tr}\left(a_{0} d a_{0}\right)^{3}$ since $\Omega^{0}=\Omega^{1}=0$. Taking into account that $a_{0}$ is a homogeneous function for $r>1$, we obtain

$$
\begin{aligned}
\operatorname{tr}\left(a_{0}^{-1} d a_{0}\right)^{3} & =\operatorname{tr}\left(m r^{-1} d r+\tilde{a}_{0}^{-1} d \tilde{a}_{0}\right)^{3} \\
& =\operatorname{tr}\left(\tilde{a}_{0}^{-1} d \tilde{a}_{0}\right)^{3}+3 m r^{-1} d r \wedge \operatorname{tr}\left(\tilde{a}_{0}^{-1} d \tilde{a}_{0}\right)^{2} \\
& \equiv 0 .
\end{aligned}
$$

Here we have put $a_{0}=r^{m} \tilde{a}_{0}$, so that $\tilde{a}_{0}$ depends on two variables and thus $\left(\tilde{a}_{0}^{-1} d \tilde{a}_{0}\right)^{3} \equiv 0$ while $\operatorname{tr}\left(\tilde{a}_{0}^{-1} d \tilde{a}_{0}\right)^{2} \equiv 0$ for any matrix-valued function $\tilde{a}_{0}$.

The second integral may be extended to all of $\partial M \times \mathbb{R}^{2}$. We would like to represent it as a trace of a formal symbol on $\partial M=\mathbb{S}^{1}$ integrated over $\tau \in \mathbb{R}$. To this end we observe that $d$ may be replaced by $d \tau \wedge \partial / \partial \tau$ everywhere in (4.6) but the last term since other differentials enter into the form $\omega=d \xi \wedge d x$. The second integral in (4.6) becomes

$$
\begin{aligned}
\frac{1}{2 \pi i} \int_{-\infty}^{\infty} d \tau \frac{1}{2 \pi} \int_{\mathbb{S}^{1} \times \mathbb{R}}\left\{\operatorname{tr}\left(r_{0} a_{0}^{\prime}\right)+\operatorname{tr}\left(r_{1} a_{0}^{\prime}-r_{0}^{\prime} a_{1}\right)\right. \\
-\frac{i}{2} \operatorname{tr}\left(\frac{\partial r_{0}}{\partial x} \frac{\partial a_{0}^{\prime}}{\partial \xi}-\frac{\partial r_{0}^{\prime}}{\partial \xi} \frac{\partial a_{0}}{\partial x}\right) \\
\left.+\frac{\partial}{\partial \tau}\left(\operatorname{tr}\left(r_{0} \frac{\partial a_{0}}{\partial \xi}\right) \xi\right)-\frac{\partial}{\partial \xi}\left(\operatorname{tr}\left(r_{0} a_{0}^{\prime}\right) \xi\right)\right\} d \xi d x
\end{aligned}
$$

The integrand may be represented as

$$
\begin{aligned}
& s(x, \xi, \tau):=\left.\operatorname{tr} \bar{r}(\tau) \circ \bar{a}^{\prime}(\tau)\right|_{1} \\
& \quad-\frac{\partial}{\partial \tau} \operatorname{tr}\left(r_{0} a_{1}\right)-\frac{i}{2} \frac{\partial}{\partial \xi} \operatorname{tr} \frac{\partial r_{0}}{\partial x} a_{0}^{\prime}+\frac{i}{2} \frac{\partial}{\partial x} \operatorname{tr} \frac{\partial r_{0}}{\partial \xi} a_{0}^{\prime}+\frac{i}{2} \frac{\partial}{\partial \tau} \operatorname{tr} \frac{\partial r_{0}}{\partial \xi} \frac{\partial a_{0}}{\partial x} \\
& +\frac{\partial}{\partial \tau}\left(\operatorname{tr}\left(r_{0} \frac{\partial a_{0}}{\partial \xi}\right) \xi\right)-\frac{\partial}{\partial \xi}\left(\operatorname{tr}\left(r_{0} a_{0}^{\prime}\right) \xi\right) .
\end{aligned}
$$

and we rewrite integral (4.7) as

$$
\frac{1}{2 \pi i} \int_{-\infty}^{\infty} d \tau \operatorname{Tr}^{\prime} \mathrm{Op} \operatorname{p}_{\partial M}(s(x, \xi, \tau))
$$

(recall that $I$ has compact support). The index formula (3.18) becomes

$$
\begin{aligned}
& \text { ind } A=\int_{S^{*} M} \operatorname{AS}(A)+\frac{1}{2 \pi i} \int_{-\infty}^{\infty} d \tau \\
& \qquad \operatorname{Tr}^{\prime}\left(\left.A_{c}^{-1}(\tau+i \gamma-i h \gamma) A_{c}^{\prime}(\tau+i \gamma-i h \gamma)\right|_{1}-\mathrm{Op} \operatorname{p}_{\partial M}\left(\left.\bar{r} \circ \bar{a}^{\prime}\right|_{1}-s\right)\right) .
\end{aligned}
$$


The symbol

$$
\begin{aligned}
\left.\bar{r}(\tau) \circ \bar{a}^{\prime}(\tau)\right|_{1}-s(\tau)= & -\frac{i}{2} \frac{\partial}{\partial x}\left(\frac{\partial r_{0}}{\partial \xi} a_{0}^{\prime}\right)+\frac{\partial}{\partial \xi}\left(r_{0} a_{0}^{\prime} \xi+\frac{i}{2} \frac{\partial r_{0}}{\partial x} a_{0}^{\prime}\right) \\
& -\frac{\partial}{\partial \tau}\left(\left(r_{0} \frac{\partial a_{0}}{\partial \xi}\right) \xi+\frac{i}{2}\left(\frac{\partial r_{0}}{\partial \xi} \frac{\partial a_{0}}{\partial x}\right)-\left(r_{0} a_{1}\right)\right)
\end{aligned}
$$

may be regarded as a new regularisation of the operator

$$
\begin{aligned}
& \left.A_{c}^{-1}(\tau+i \gamma-i h \gamma) A_{c}^{\prime}(\tau+i \gamma-i h \gamma)\right|_{1} \\
& \quad=A_{c}^{-1}(\tau+i \gamma) A_{c}^{\prime}(\tau+i \gamma)-i \gamma \frac{d}{d \tau}\left(A_{c}^{-1}(\tau+i \gamma) A_{c}^{\prime}(\tau+i \gamma)\right) .
\end{aligned}
$$

This new regularisation is in a sense canonical since the behaviour of the symbols $\bar{a}$ and $\bar{r}$ inside the ball $r<1$ does not affect the result. This property may be seen directly from (4.9) because all the terms involved are complete derivatives.

In Remark 3.5, we have introduced another canonical regularisation given by the functional $\overline{\mathrm{Tr}}$. The two regularisations differ by

$$
\frac{1}{2 \pi i} \overline{\operatorname{Tr}} \mathrm{Op} \operatorname{p}_{\partial M}\left(s-\left.\bar{r} \circ \bar{a}^{\prime}\right|_{1}\right)
$$

and this expression may be computed easily using (4.9). The terms of the form $(\partial / \partial \xi)(\cdot)$ or $(\partial / \partial x)(\cdot)$ are annihilated by $\overline{\operatorname{Tr}}$ while $\overline{\operatorname{Tr}}(\partial / \partial \tau)(\cdot)$ can be computed by Proposition 6 of [Me195]. In the notation of [Me195],

$$
\begin{aligned}
\frac{1}{2 \pi i} & \overline{\operatorname{Tr}} \mathrm{Op}_{\partial M}\left(s-\left.\bar{r} \circ \bar{a}^{\prime}\right|_{1}\right) \\
& =-\frac{1}{2 \pi i} \widetilde{\operatorname{Tr}} \mathrm{Op} \operatorname{p}_{\partial M}\left(\operatorname{tr}\left(r_{0} \frac{\partial}{\partial \xi}\right) \xi+\frac{i}{2} \operatorname{tr} \frac{\partial r_{0}}{\partial \xi} \frac{\partial a_{0}}{\partial x}-\operatorname{tr} r_{0} a_{1}\right) \\
& =-\left.\frac{1}{4 \pi^{2}} \int_{\mathbb{S} 1 \times \mathbb{R}}\left(\frac{1}{2} \operatorname{tr} \frac{\partial r_{0}}{\partial \xi} \frac{\partial a_{0}}{\partial x}+i \operatorname{tr} r_{0} a_{1}\right)\right|_{\tau=-1} ^{\tau=1} d \xi d x
\end{aligned}
$$

For further references let us write down the index formula for $n=2$ in detail,

$$
\text { ind } \begin{aligned}
A & =\frac{1}{4 \pi^{2}} \int_{S^{*} M} \frac{1}{6} \operatorname{tr}\left(a_{0}^{-1} \partial a_{0}\right)^{3}-\frac{1}{2} \operatorname{tr}\left(a_{0}^{-1} \Omega^{1} \wedge \partial a_{0}+a_{0}^{-1} \partial a_{0} \Omega^{0}\right) \\
& +\frac{1}{2 \pi i} \overline{T r}\left(A_{c}^{-1}(\tau+i \gamma) A_{c}^{\prime}(\tau+i \gamma)-i \gamma \frac{d}{d \tau}\left(A_{c}^{-1}(\tau+i \gamma) A_{c}^{\prime}(\tau+i \gamma)\right)\right) \\
& -\left.\frac{1}{4 \pi^{2}} \int_{\mathbb{S}^{1} \times \mathbb{R}}\left(\frac{1}{2} \operatorname{tr}\left(a_{0}^{-1} \frac{\partial a_{0}}{\partial \xi} a_{0}^{-1} \frac{\partial a_{0}}{\partial x}\right)+i \operatorname{tr} a_{0}^{-1} a_{1}\right)\right|_{\tau=-1} ^{\tau=1} d \xi d x
\end{aligned}
$$

Remark 4.2 Note that formula (4.10) being homotopy invariant implies that it extends to all symbols $a(t, \tau)$ exponentially stabilising when $t \rightarrow \infty$. In this case the boundary values in $(4.10)$ are $a(\tau, \infty)$. 


\section{$5 \quad$ Examples}

In this section we consider the Cauchy-Riemann operator on a complex plane $\mathbb{C}$ treating the point at infinity as a conical point. Even for this simple operator the additional term in (4.10) may be non-trivial. Although all the bundles are trivial over $\mathbb{C}$, the trivialisations and connections may be chosen in different ways. Our goal is to observe how these choices affect the three terms in (4.10). So, we consider two cases:

1. the Cauchy-Riemann operator from functions to (0,1)-forms,

$$
\bar{\partial}: u \mapsto d \bar{z} \frac{\partial u}{\partial \bar{z}}
$$

2. the Cauchy-Riemann operator from functions to functions,

$$
\frac{\partial}{\partial \bar{z}}: u \mapsto \frac{\partial u}{\partial \bar{z}}
$$

Near $z=\infty$ we introduce new coordinates

$$
\zeta=t+i \varphi, \quad t \in \mathbb{R}_{+}, \varphi \in \mathbb{R}(\bmod 2 \pi),
$$

setting $z=e^{\zeta}$

Case 1.

We take the global frame 1 for $E^{0}$ and $d \bar{z}, d \bar{\zeta}$ as local frames in $U_{0}=$ $\{|z|<2\}$ and $U_{\infty}=\{\Re \zeta>0\}$ for $E^{1}$. In these frames we have

$$
\begin{aligned}
A & =\frac{\partial}{\partial x}+i \frac{\partial}{\partial y}, \\
a_{0} & =i(\xi+i \eta)
\end{aligned}
$$

in $U_{0}$ and

$$
\begin{aligned}
A & =\frac{\partial}{\partial t}+i \frac{\partial}{\partial \varphi} \\
a_{0} & =i(\tau+i \sigma), \\
A_{c}(\tau) & =i\left(\tau+\frac{\partial}{\partial \varphi}\right)
\end{aligned}
$$

in $U_{\infty}$. Thus,

$$
A_{c}^{-1}(\tau) A_{c}^{\prime}(\tau)=\left(\tau+\frac{\partial}{\partial \varphi}\right)^{-1}
$$

Case 2.

We take again the global frame 1 for $E^{0}$ and $1, e^{-t}$ as local frames in $U_{0}$ and $U_{\infty}$ for $E^{1} \cong E^{0}$. Again we have

$$
\begin{aligned}
A & =\frac{\partial}{\partial x}+i \frac{\partial}{\partial y}, \\
a_{0} & =i(\xi+i \eta)
\end{aligned}
$$


in $U_{0}$, but

$$
\begin{aligned}
A & =e^{i \varphi}\left(\frac{\partial}{\partial t}+i \frac{\partial}{\partial \varphi}\right), \\
a_{0} & =i e^{i \varphi}(\tau+i \sigma), \\
A_{c}(\tau) & =i e^{i \varphi}\left(\tau+\frac{\partial}{\partial \varphi}\right)
\end{aligned}
$$

in $U_{\infty}$. Then, just as in Case 1 ,

$$
A_{c}^{-1}(\tau) A_{c}^{\prime}(\tau)=\left(\tau+\frac{\partial}{\partial \varphi}\right)^{-1}
$$

and so in both cases the $\eta$-invariant term is the same.

The functions $e^{i k \varphi}, k \in \mathbb{Z}$, form an orthonormal basis of eigenfunctions of (5.1) with eigenvalues

$$
\lambda_{k}(\tau)=(\tau+i k)^{-1},
$$

provided that $\tau \neq-i k$ for $k \in \mathbb{Z}$. The conormal ellipticity holds for weight lines $\Gamma_{\gamma}$ with $\gamma \notin \mathbb{Z}$.

To compute $\overline{\operatorname{Tr}} A_{c}^{-1} A_{c}^{\prime}$ consider

$$
\begin{aligned}
\operatorname{Tr}^{\prime} \frac{d}{d \tau}\left(A_{c}^{-1}(\tau) A_{c}^{\prime}(\tau)\right) & =-\sum_{k=-\infty}^{\infty}(\tau+i k)^{-2} \\
& =\pi \frac{d}{d \tau} \operatorname{coth} \pi \tau
\end{aligned}
$$

The first integration gives $\pi \operatorname{coth} \pi \tau$. Next, if $\gamma<0$, then

$$
\begin{aligned}
\overline{\operatorname{Tr}}\left(A_{c}^{-1}(\tau+i \gamma) A_{c}^{\prime}(\tau+i \gamma)\right) & =\lim _{T \rightarrow \infty} \int_{-T}^{T} \pi \operatorname{coth} \pi(\tau+i \gamma) d \tau \\
& =\lim _{T \rightarrow \infty} \int_{-\pi T+i \pi \gamma}^{\pi T+i \pi \gamma} \operatorname{coth} z d z \\
& =-2 \pi i n-\pi i+2 \pi i \gamma
\end{aligned}
$$

where $n$ is the number of poles of $\operatorname{coth} z$ in the $\operatorname{strip} \Im z \in(0, \pi \gamma)$. This may be seen by integrating over the contour which is the boundary of the rectangle with vertices $-\pi T, \pi T,-\pi T+i \pi \gamma, \pi T+i \pi \gamma$ and with removed small disc centered at the origin. The principal value of the integral over the interval $(-\pi T, \pi T)$ vanishes since $\operatorname{coth} z$ is an odd function.

The second term under $\overline{\mathrm{Tr}}$ functional gives

$$
\begin{aligned}
\overline{\operatorname{Tr}}\left(-i \gamma \frac{d}{d \tau}\left(A_{c}^{-1}(\tau+i \gamma) A_{c}^{\prime}(\tau+i \gamma)\right)\right) & =-i \gamma \int_{-\infty}^{\infty} \frac{d}{d \tau} \pi \operatorname{coth} \pi(\tau+i \gamma) d \tau \\
& =-2 \pi i \gamma
\end{aligned}
$$


Thus, the whole $\eta$-invariant term is equal to $-\left(n+\frac{1}{2}\right)$, for $\gamma<0$. In general, this term is equal to $-\left(n+\frac{1}{2}\right) \operatorname{sgn} \gamma$.

Consider now the interior term. The form $\operatorname{tr}\left(a_{0}^{-1} \partial a_{0}\right)^{3}$ vanishes identically since $a_{0}^{-1} \partial a$ is a scalar 1 -form. Thus, the interior term vanishes in Case 2 since $\Omega^{0}=\Omega^{1}=0$ in this case.

In Case 1 the interior term is

$$
-\frac{1}{8 \pi^{2}} \int_{S^{*} M} \frac{d(\xi+i \eta)}{\xi+i \eta} \Omega^{1}
$$

where the orientation is defined by the form $d \xi \wedge d x \wedge d \eta \wedge d y$ on $T^{*} M$. We first change the orientation form to $d \xi \wedge d \eta \wedge d x \wedge d y$ and then compute the integral of the first factor in (5.2) obtaining

$$
\frac{i}{4 \pi} \int_{M} \Omega^{1}
$$

with the orientation form $d x \wedge d y=d t \wedge d \varphi$. Introduce connection forms $\Gamma_{0}^{1}$, $\Gamma_{\infty}^{1}$ in $U_{0}, U_{\infty}$ for the bundle $E^{1}=\Lambda^{0,1}$, i.e., define covariant differentials of the frames $d \bar{z}$ in $U_{0}$ and $d \bar{\zeta}$ in $U_{\infty}$. In $U_{0}$ we set

$$
\begin{aligned}
\partial(d \bar{z}) & :=\left(d \rho_{0}\right) d \bar{z}+d\left(\rho_{\infty} e^{\bar{\zeta}}\right) d \bar{\zeta} \\
& =\left(d \rho_{0}+d\left(\rho_{\infty} e^{\bar{\zeta}}\right) e^{-\bar{\zeta}}\right) d \bar{z}
\end{aligned}
$$

so that

$$
\begin{aligned}
\Gamma_{0}^{1} & =d \rho_{0}+d\left(\rho_{\infty} e^{\bar{\zeta}}\right) e^{-\bar{\zeta}} \\
& =\rho_{\infty} d \bar{\zeta}
\end{aligned}
$$

Similarly, $\Gamma_{\infty}^{1}=-\rho_{0} d \bar{\zeta}$.

It is a simple matter to see that these forms satisfy the usual transition rule for connection coefficients. Then

$$
\begin{aligned}
\Omega^{1} & =d \gamma_{0}^{1} \\
& =d \gamma_{\infty}^{1} \\
& =d \rho_{\infty} \wedge d \bar{\zeta} \\
& =-i d \rho_{\infty} \wedge d \varphi
\end{aligned}
$$

whence

$$
\frac{i}{4 \pi} \int_{M} \Omega^{1}=\frac{1}{2}
$$

Finally, let us compute the additional term in (4.10). The lower order symbol $a_{1}$ vanishes in both cases. Moreover, in Case 1 the whole additional term is zero, since $a_{0}$ does not depend on $x$. 
For Case 2 we have

$$
\begin{aligned}
-\left.\frac{1}{4 \pi^{2}} \int_{\mathbb{S} 1} \int_{-\infty}^{\infty}\left(-\frac{1}{2}\right) \frac{1}{\tau+i \sigma}\right|_{\tau=-1} ^{\tau=1} d \sigma d \varphi & =\frac{1}{4 \pi^{2}} \int_{0}^{2 \pi} d \varphi \int_{-\infty}^{\infty} \frac{1}{1+\sigma^{2}} d \sigma \\
& =\frac{1}{2} .
\end{aligned}
$$

So, we obtain

$$
\text { ind } \begin{aligned}
\bar{\partial} & =\operatorname{ind} \frac{\partial}{\partial \bar{z}} \\
& =\frac{1}{2}-\left(n+\frac{1}{2}\right) \operatorname{sgn} \gamma .
\end{aligned}
$$

For $\gamma<0$ one can easily recognise the classical Liouville theorem for entire functions. Indeed, the inclusion $u \in H^{s, \gamma}$ with $\gamma<0$ implies that $|u| \leq C e^{-\gamma t}$ as $t \rightarrow \infty$, or $|u| \leq C|z|^{-\gamma}$. If $u \in \operatorname{ker} \bar{\partial}$, then, by the Liouville theorem, it is a polynomial of degree not greater than $[-\gamma]$, and $\operatorname{dim} \operatorname{ker} \bar{\partial}=[-\gamma]+1$. The cokernel in this case is empty (as is always the case for differential operators and $\gamma<0$ ). 


\section{References}

[Ang93] N. Anghel, An abstract index theorem on non-compact Riemannian manifolds, Houston J. Math. 19 (1993), 223-237.

[APS75] M. F. Atiyah, V. K. Patodi, and I. M. Singer, Spectral asymmetry and Riemannian geometry. I, Math. Proc. Camb. Phil. Soc. 77 (1975), 43-69.

[BC89] M. Bismut and J. Cheeger, Eta-invariants and their adiabatic limits, J.A.M.S. 2 (1989), 33-70.

[BC90] M. Bismut and J. Cheeger, Families index for manifolds with boundary, superconnections and cones, Invent. Math. 89 (1990), 91-151.

[BG92] Th. P. Branson and P. B. Gilkey, Residues of the eta function for an operator of Dirac type, J. Funct. Anal. 108 (1992), no. 1, $47-87$.

[Brü90] J. Brüning, $L^{2}$-index theorems on certain complete manifolds, J. Differential Geom. 32 (1990), no. 2, 491-532.

[BS88] J. Brüning and R. Seeley, An index theorem for first order regular singular operators, Amer. J. Math. 110 (1988), no. 4, 659-714.

[Che83] J. Cheeger, Spectral geometry of singular Riemannian spaces, J. Differential Geom. 18 (1983), 175-221.

[Che87] J. Cheeger, Eta invariants, the adiabatic approximation and conical singularities, J. Differential Geom. 26 (1987), 175-221.

[Cho85] A. W. Chou, The Dirac operator on spaces with conical singularities and positive scalar curvatures, Trans. Amer. Math. Soc. 289 (1985), no. 1, 1-40.

[DF 83] H. Donnelly and C. Fefferman, $L^{2}$-cohomology and index theorem for the Bergman metric, Ann. of Math. 118 (1983), no. 3, 593618.

[DW91] R. G. Douglas and K. P. Wojciechowski, Adiabatic limits of the eta-invariants. The odd-dimensional Atiyah-Patodi-Singer problem, Comm. Math. Phys. 142 (1991), 139-168.

[Fed74] B. V. Fedosov, Analytic formulas for the index of elliptic operators, Trans Moscow Math. Soc. 30 (1974), 159-241. 
[FS96] B. V. Fedosov and B.-W. Schulze, On the index of elliptic operators on a cone, Schrödinger Operators, Markov Semigroups, Wavelet Analysis, Operator Algebras, Advances in Partial Differential Equations, vol. 3, Akademie-Verlag, Berlin, 1996, pp. 347372.

[FST96] B. V. Fedosov, B.-W. Schulze, and N. N. Tarkhanov, On the Index of Elliptic Operators on a Wedge, Preprint MPI/96-143, Max-Planck-Inst. für Math., Bonn, 1996, 46 pp.

[Get93] E. Getzler, Cyclic homology and the Atiyah-Patodi-Singer index theorem, Contemp. Math. 148 (1993), 19-45.

[GL83] M. Gromov and H. B. Lawson, Positive scalar curvature and the Dirac operator on complete Riemannian manifolds, Publ. Math. I.H.E.S. 58 (1983), 83-196.

[Gru92] G. Grubb, Heat operator trace expansions and index for general Atiyah-Patodi-Singer boundary problems, Comm. Part. Diff. Equ. 17 (1992), 2031-2077.

[GS95] G. Grubb and R. Seeley, Weakly parametric pseudodifferential operators and Atiyah-Patodi-Singer boundary problems, Invent. Math. 121 (1995), 481-529.

[HMM96] A. Hassel, R. Mazzeo, and R. B. Melrose, A signature formula for manifolds with corners of codimension two, Preprint, MIT, Boston, September 1996 (to appear in Topology).

[KW93] S. Klimek and K. Wojciechowski, $\eta$-invariants on manifolds with cylindrical ends, Differential Geom. Appl. 3 (1993), 191-201.

[Les97] M. Lesch, Operators of Fuchs Type, Conical Singularities, and Asymptotic Methods, Teubner-Texte zur Mathematik, vol. 136, B.G. Teubner Verlagsgesellschaft, Stuttgart-Leipzig, 1997.

[Lot92] J. Lott, Higher eta-invariants, K-Theory 6 (1992), 191-233.

[Mel81] R. B. Melrose, Transformation of boundary problems, Acta Math. 147 (1981), no. 3-4, 149-236.

[Me193] R. B. Melrose, The Atiyah-Patodi-Singer Index Theorem, A K Peters, Wellesley, Mass, 1993.

[Me195] R. B. Melrose, The eta invariant and families of pseudodifferential operators, Math. Res. Letters 2 (1995), no. 5, 541-561.

[MM83] R. B. Melrose and G. A. Mendoza, Elliptic Operators of Totally Characteristic Type, Preprint MSRI 047-83, MIT, Berkeley, 1983. 
[MN96a] R. B. Melrose and V. Nistor, Homology of Pseudodifferential Operators. I: Manifolds with Boundary, Penn State Preprint, May 1996.

[MN96b] R. B. Melrose and V. Nistor, The $\mathbb{R}^{k}$-Equivariant Index Theorem and $C^{*}$-Algebras of b-Pseudodifferential Operators, Penn State Preprint, November 1996 (to appear in GAFA).

[MP93] R. B. Melrose and P. Piazza, Families of Dirac Operators, Boundaries and the b-Calculus, Preprint, MIT, Boston, 1993.

[Mü187] W. Müller, Manifolds with cusps of rank one, spectral theory and $L^{2}$-index theorem, Lect. Notes Math., vol. 1244, Springer-Verlag, Berlin, 1987.

[Mü194] W. Müller, Eta invariants and manifolds with boundary, J. Diff. Geom. 40 (1994), 311-377.

[Pia93] P. Piazza, On the index of elliptic operators on manifolds with boundary, J. Funct. Anal. 117 (1993), 308-359.

[PR90] B. A. Plamenevskii and G. V. Rozenblum, On the index of pseudodifferential operators with isolated singularities in symbols, A1gebra i Analiz 2 (1990), no. 5, 165-188 (Russian).

[PR92] B. A. Plamenevskii and G. V. Rozenblum, Pseudodifferential operators with discontinuous symbols: $K$-theory and index formulas, Funkts. Analiz 26 (1992), 266-275 (Russian).

[Roz94] G. Rozenblum, Index Formulae for Pseudodifferential Operators with Discontinuous Symbols, Preprint 32, Kobenhavns Univ., Copenhagen, December 1994.

[Roz96] G. Rozenblum, The Index of Cone Mellin Operators, Preprint, Göteborg Univ., Göteborg, April 1996.

[Sch94] B.-W. Schulze, Pseudo-Differential Boundary Value Problems, Conical Singularities, and Asymptotics, Akademie-Verlag, Berlin, 1994.

[SSS97] B.-W. Schulze, B. Sternin, and V. Shatalov, On the Index of Differential Operators on Manifolds with Conical Singularities, Preprint 97/10, Univ. Potsdam, Potsdam, May 1997.

[ST97] B.-W. Schulze and N. N. Tarkhanov, The index of elliptic operators on manifolds with cusps, Differential Equations, Asymptotic Analysis, and Mathematical Physics, Math. Research, vol. 100, Akademie-Verlag, Berlin, 1997, pp. 344-359. 
[Ste89] M. Stern, $L^{2}$-index theorems on locally symmetric spaces, Invent. Math. 96 (1989), 231-282.

[Ste90] M. Stern, Eta invariants and Hermitian locally symmetric spaces, J. Differential Geom. 31 (1990), 771-789.

[Wu93] F. Wu, The Chern character and the eta-invariant of the Dirac operator, K-Theory 7 (1993), 145-174. 